\title{
A practical codification and its analysis for the generalized reconfiguration problem
}

\author{
Carlos Henrique Nogueira de Resende Barbosa ${ }^{\mathrm{a}, \mathrm{b}, *}$, Rafael Frederico Alexandre ${ }^{\mathrm{a}, \mathrm{b}}$, \\ João Antônio de Vasconcelos ${ }^{\mathrm{b}}$ \\ a Federal University of Ouro Preto, Departamento de Ciências Exatas e Aplicadas, Rua 37, no. 115, Loanda, CEP: 35931-026 - João Monlevade, MG, Brazil \\ ${ }^{\mathrm{b}}$ Federal University of Minas Gerais, Graduate Program in Electrical Engineering, Av. Antônio Carlos, 6627 - Pampulha, CEP: $31270-901$ - Belo Horizonte, MG, Brazil
}

\section{A R T I C L E I N F O}

Article history:

Received 20 May 2012

Received in revised form 6 October 2012

Accepted 3 December 2012

Available online 16 January 2013

Keywords:

Radial distribution networks

Multi-objective

Constrained optimization problem

Graph theory

\begin{abstract}
A B S T R A C T
Distribution network reconfiguration problem is simply aimed at finding the best set of radial configurations among a huge number of possibilities. Each solution of this set ensures optimal operation of the system without violating any prescribed constraint. To solve such problem, it is important to count on efficient procedure to enforce radiality. In this paper, we propose a reliable approach to deal properly with topology constraint enabling algorithm convergence toward optimal or quasi-optimal solutions. A simple and practical codification of individuals used in evolutionary algorithms to solve the generalized reconfiguration problem is detailed in this paper. Mathematical formulation, algorithm, and simulation results are presented for the distribution reconfiguration problem, incorporating a new representation scheme which is immune to topologically unfeasible possibilities. The individual interpretation procedure is straight and it demands no additional data structure or graph preprocessing. Comparisons are made for five well-known distribution systems to demonstrate the efficacy of the proposed methodology. It is also demonstrated that optimal configurations are properly surveyed when single or multiple sources are dealt.
\end{abstract}

(C) 2012 Elsevier B.V. All rights reserved.

\section{Introduction}

Reconfiguration is often employed in distribution networks to improve their operating conditions which are guided by common theoretical indexes such as power loss and voltage deviation. As a matter of concern, reconfiguration of such networks can be done only if system security is preserved. The distribution feeder reconfiguration, known as the DFR problem in short, is understood as altering the interconnections between busses by means of switching on and off the connecting lines. As stated by Zhu [1], DFR optimization problem consists in finding feasible configurations over a meshed structure which may be able to improve objectives as much as possible while operating constraints are satisfied [1]. In this sense, the radial topology is preferred over others due to the coordination of protective devices at lower financial costs and lesser vulnerability against overcurrent. The DFR problem is

\footnotetext{
* Corresponding author at: Federal University of Ouro Preto, Departamento de Ciências Exatas e Aplicadas, Rua 37, no. 115, Loanda, CEP: 35931-026 - João Monlevade, MG, Brazil. Tel.: +55 31 38528709; fax: +55 3138528709 .

E-mail addresses: carloshnrb@gmail.com, cbarbosa@decea.ufop.br (C.H.N.d.R. Barbosa), rfalexandre@gmail.com (R.F. Alexandre), jvasconcelos@ufmg.br (J.A.d. Vasconcelos).
}

highly combinatorial since the number of possible configurations increases exponentially according to the number of $N_{W}$ maneuverable switches (sectionalizing and tie-switches included) in the distribution network $[2,3]$. Due to it, feasible configurations set belong to a small fraction of the space delimited by topological and electrical constraints which is a challenge for most of the searching methods.

The DFR problem is characterized as being a multimodal and combinatorial optimization problem, generally described by a set of nonlinear and non-differentiable functions. Being a NP-complete problem [4,5], there is no known method able to provide exact solution within a reasonable time. Its complexity weakens the effectiveness of any pre-established guideline unless the approach is restricted to particular network structure, as expert systems are. Exact solution (or optima set) is obtained by sweeping the entire search space to find every feasible configuration. This exhaustive procedure requires intensive computing to evaluate $2^{N_{W}}$ combinations. In real-world distribution networks, maneuverable switches are numerous and the computational effort spent to find the pareto-optimal solutions (PoS) becomes prohibitive as we will demonstrate in this paper. Despite of problem dimensionality, larger search spaces implicate on more opportunities for greater losses reduction and better system operating conditions probed by means of reconfiguration [6]. 
One of the main challenges closely related to the discrete and combinatorial nature of DFR problem is the representation of solutions. In this article, codification is our main concern and a complete scheme to represent, interpret and recombine solutions is described. As stated in [7,8], the individual representation determines the success or failure of the optimization procedure tackled by any evolutionary algorithm. This paper keeps its focus on a novel, simple and generic, but not less efficient, individual codification that needs no additional computation to repair individuals or special genetic operator customization. Our strategy works for single and multiple sources as well as for networks where some of the lines have no switches. From the experiments, we have clear evidence that our scheme does not disturb the convergence of the evolutionary algorithm. It trusts on a proper individual interpretation which is done gradually to avoid topology violation such as bus islanding or loop. The way the individual is built and modified satisfies many properties required for encoding techniques reported by Raidl [9], such as feasibility and bias. The decoding procedure has lower complexity keeping the search space coverage at a reasonable level. Although we have applied our codification scheme to an NSGA-II [10] implementation, it is also adaptable to any other population-based meta-heuristic search technique.

This paper is organized as follows. In Section 2, related works are discussed and their main drawbacks and specialties are commented. The restricted multi-objective formulation to the feeder reconfiguration problem is given in Section 3. In Section 4, details about our codification scheme and associated stochastic operators are provided justifying the usefulness of the proposed methodology. Finally, experimental results are shown in Section 5 while concluding remarks of this work are summarized in Section 6 .

\section{Previous works}

In the last three decades, much work has been dedicated to improve methodologies and adapt the algorithms which deal with DFR optimization problem, making them proper, faster and more robust in searching for global optima, especially when large-sized distribution networks are dealt. Initially, heuristic techniques were thought to be the best option due to the combinatorial nature of DFR problem. Many proposals as the methods reviewed by Sarfi [11] range from pure heuristics [6,12-14] to blended methods [15-17]. They were widely employed in the past to solve DFR problem and they are still being used [18-21]. Nonetheless, those methods are susceptible to landscape entrapments and regions of attraction created by local optima. To overcome such drawbacks, evolutionary algorithms have been a promising class of techniques in solving combinatorial problems satisfactorily. Although these techniques were first introduced to the DFR problem by Nara using classical genetic algorithms in the 1980s [22], the performance of such algorithms has been hardly affected by the chromosome representation ever since $[23,24]$. Since then, several skillful codification schemes have already been proposed by researchers in order to properly deal with topology check which is considered the main hurdle of the DFR problem [25]. On binary coding, bit 0 (1) conventionally denotes an open (closed) switch of the distribution network $[22,26]$. From technical viewpoint, codification schemes have been classified in two main types: node encoding and branch encoding. Of course, secondary encoding strategies conceived as a combination of the two previous codifications have also been proposed like path-to-node [27], matroid theory, [28] and network random key [7].

This brief review focused on the main contributions of codification applied to meta-heuristic techniques found in the specialized literature. Particularly, our codification scheme is derived from Carreno's proposal [29]. In that work, he presented a branch encoding approach which aimed on better application of stochastic operators. Its chromosome contained $N_{L}$ genes which were grouped into two parts to represent active and inactive lines. A time-consuming local search was employed in place of mutation operator. This local improvement step worked as a limited brute force trying all possible combination derived from the best solution known hitherto. To efficiently implement it, knowledge about loops and paths to substation were required in advance at the expense of additional processing. Carreno's strategy may degrade diversity on the current population leading to local optima. His integer codification has followed previous schemes based on strings, presented by Hsiao [23], Shin [30], Huang [31], and Kumar [32]. Although being easy to implement, those strings (characterisc vectors) are longer as the network size increases. Aiming at more concise representations, Zhu [1] coded only the open switches of a distribution network. The suggestions came from Zhu brought some efficiency to the representation proposed by Nara [22] to the landscape searching and to the algorithm itself with the shortening of chromosome length. Other simpler node encoding representations adopted integer codification focusing more on the content of the individual than on the stochastic operator functionalities. To cite a few works, predecessor node [33] and predecessor node with restricted alphabet [34] could be pointed out. Thus, those strategies soften the individual interpretation but feasibility assessment becomes burdened. Milani [35] also employed a chromosome structure dividing genes into groups of substrings to represent, in a binary form, the set of branches pertaining to the same loop. To improve the search throughout the viable space, he adopted a scattered crossover and mutation with variable rate as genetic operators. Adopting a variable length differential vector, Lin [36] coded only switches whose status were modified. As an alternative, Lin also proposed a partial non-random initial population and a probability rate shared by both crossover and mutation operators to enable balance between exploration and exploitation fed back by the actual performance of the algorithm. Following a quite different strategy, Mendoza [37] also used an integer codification to enumerate open switches in the network. His work was primarily based on graph/topological analysis. As a consequence, his strategy demanded preprocessing to generate a lookup table of fundamental loops. Any further description about repair mechanism was not provided which could be probably based on hit and trial. Swarnkar [25] conceived an individual encoding, as Mendoza did, whose application was guided by a set of rules along with concepts on graph theory. In that work, the optimization process should start up from an initial solution found by a branch-exchange heuristic [13]. As in [37], this strategy demanded supplemental information about the system under analysis. It is noteworthy that this preprocessing stage is much costly for meshed and larger structures. Furthermore, the rate of successful crossovers decreases with the number of loops in the network [28]. Even adopting a similar chromosome structure to Carreno's proposal, our representation scheme is submitted to a different interpretation which is the key to ensure immunity to unfeasible solutions that would be produced by an evolutionary algorithm (EA). In our proposition, there is no effort applied to avoid or repair unfeasible offspring, since we simply circumvent these obstacles by means of a flexible interpretation. Avoiding most of the drawbacks seen in the references above is our main concern.

\section{DFR mathematical model}

Once DFR optimization problem is closely related to configuration, optimization variables should reflect somehow the interconnections among busses. Commonly, any configuration $c$ can be described by on/off statuses of the $N_{W}$ switches. A binary vector $\boldsymbol{s} \boldsymbol{w}^{\boldsymbol{c}}\left(\boldsymbol{s} \boldsymbol{w}^{\boldsymbol{c}}=\left[s w_{1}^{c} \ldots s w_{q}^{c} \ldots s w_{N_{W}}^{c}\right]\right)$ is a possible representation 
to the network where the $q$ th element indicates whether the corresponding switch is open $\left(s w_{q}^{c}=0\right)$ or closed $\left(s w_{q}^{c}=1\right)$, regardless its type (tie or sectionalizing). Instead of using a conventional binary vector, an integer vector is employed to code a configuration that will be interpreted in a sequential mode. We represented this same integer vector by the equivalent binary type to allow a systematic analysis of our proposition described in our mathematical model below. The objective functions for DFR problem are, in general, nonlinear and non-continuous with several local optima [38]. Some authors [8,31] have already been treating multi-objective formulation for the DFR problem as a combination of the objectives into one single weighted function. As a consequence, actual non-dominated solutions may not have been attained. Here, we present the most common criteria employed on reconfiguration cases along with critical constraints.

\subsection{Objectives}

In the multi-objective formulation presented here, the real power losses $(P L)$ have to be minimized:

$P L(\boldsymbol{s w})=\sum_{i \in \mathbf{\Omega}, q \in \boldsymbol{W}} s w_{q}^{c} \cdot \operatorname{Re}\left(Z_{i}\right) \cdot\left|I_{i}\right|^{2}+\sum_{i \in \boldsymbol{\Psi}} \operatorname{Re}\left(Z_{i}\right) \cdot\left|I_{i}\right|^{2}$

in which $\boldsymbol{\Omega} \subset \boldsymbol{L}$ and $\boldsymbol{W} \equiv \boldsymbol{\omega} \cup \overline{\boldsymbol{\omega}}$. For multiple source systems, total power losses are obtained from the summation of partial power losses associated with each feeder, as stated in [3]. It is also important to keep bus voltage profile as flat as possible, represented by the voltage deviation index (VDI) and defined as in [31]:

$V D I(\boldsymbol{s w})=\frac{1}{N_{B}} \vartheta\left(k, \boldsymbol{s w}^{c}\right) \cdot\left|V_{k}-V_{k}^{n o m}\right|$

wherein $\vartheta\left(k, \boldsymbol{s} \boldsymbol{w}^{c}\right)$ denotes a boolean function indicating if bus is energized $\left(\vartheta\left(k, \boldsymbol{s} \boldsymbol{w}^{c}\right)=1\right)$ or not $\left(\vartheta\left(k, \boldsymbol{s} \boldsymbol{w}^{c}\right)=0\right)$ according to the statuses of the $N_{W}$ switches. This criterion was explicitly written in terms of decision variables $s w_{q}^{c}$ to emphasize its interdependence with those variables. For multiple source systems, an averaged VDI can be calculated:

$\overline{V D I}=\frac{1}{N_{R}} \cdot \sum_{t=1}^{N_{R}} V D I_{t}$

The next criterion is the Current Loading Index (CLI) responsible for loading equalization across the network in order to reach the minimal acceptable current flowing through all lines. Thus, smaller the value better is the configuration, defined as [31]:

$C L I(\mathbf{s w})=\frac{1}{N_{L}} \cdot\left[\sum_{i \in \boldsymbol{\Omega}, q \in \boldsymbol{W}} s w_{q}^{c} \cdot \frac{I_{i}}{I_{i}^{\max }}+\sum_{i \in \boldsymbol{\Psi}} \frac{I_{i}}{I_{i}^{\max }}\right]$

Similarly, CLI for multiple sources is calculated considering every line in the system. From the viewpoint of practical configuration attainment, it is important to consider time feasibility to perform maneuvers. Thus, the inter-distance between initial and targeted configurations is a reasonable measure to indicate migration complexity. So, this criterion named number of switching (NS) needs to be minimized also:

$N S(\mathbf{s} \boldsymbol{w})=\boldsymbol{s} \boldsymbol{w}^{\boldsymbol{c}}-\mathbf{s} \boldsymbol{w}^{\mathbf{0}}=\sum_{q \in W}\left|s w_{q}^{c}-s w_{q}^{0}\right|$

wherein $\boldsymbol{s} \boldsymbol{w}^{\boldsymbol{c}}$ and $\boldsymbol{s} \boldsymbol{w}^{\mathbf{0}}$ stand for switch status vectors corresponding to the candidate and initial (referenced) configurations, respectively. Extension to multiple sources is straightforward because all $N_{W}$ switches are taken into consideration. Several works pointed out that DFR optima set should be found regardless the initial configuration of the feeders $[6,13,17]$, but final solution set is surely affected by $N S$, since it restricts switching.

\subsection{Equality and inequality constraints}

For a more realistic approach, several constraints must be added to the restricted version of the DFR problem. Some of these restrictions cannot be straight coded into individuals and thus violation must be evaluated while fitness is computed as usual. Other constraints can be easily incorporated into individuals due to codification. According to Lopez [39] and Mendoza [37], radiality constraint can be satisfactorily described by:

$N_{B}-N_{R}=\|\boldsymbol{\tau}\|+\|\boldsymbol{\Psi}\|$

in which $\boldsymbol{\tau}$ represents the set of powered lines such that $\boldsymbol{\tau}=\{i$ : $\left.\exists ! q, s w_{q}^{c}=1 \Rightarrow q \cup \omega \Leftrightarrow i \cup \boldsymbol{\tau}, \overline{\boldsymbol{\tau}} \backslash i, \forall i \in \boldsymbol{\Omega}\right\}$. Although needed, Eq. (6) is not enough for multiple sources. A more complete description is given as follows:

$\|\boldsymbol{\tau}\|+\|\boldsymbol{\Psi}\|=N_{L}-\|\bar{\tau}\|=N_{B}-N_{R}$

$\|\overline{\boldsymbol{\tau}}\|=N_{L}=N_{L}-N_{B}+N_{R}$

wherein $\overline{\boldsymbol{\tau}}=\left\{i: \exists ! q, s w_{q}^{c}=0 \Rightarrow q \cup \overline{\boldsymbol{\omega}} \Leftrightarrow i \cup \overline{\boldsymbol{\tau}}, \boldsymbol{\tau} \backslash i, \forall i \in \boldsymbol{\Omega}\right\} \quad$ stands for the open switches set and $N_{R}$ denotes the number of trees (NT) to be obtained for an overall feasible configuration. For topology restriction, the single path condition must ensure that every load bus in the system can only be reached by one single source through a unique path. In this sense, the supplementary equations are given below:

$\sum_{r=1}^{N_{R}} \eta\left(k, r, \boldsymbol{s} \boldsymbol{w}^{c}\right)=1, \quad \forall k=1, \ldots, N_{B}$

wherein $\eta$ is a boolean function returning one (zero) to indicate that bus $k$ is fed (not fed) by source $r$. The status of switch $s w_{q}^{c}$ which delivers power to $k$ th bus depends on the status of the next upstream switch $s w_{q-1}^{c}$ :

$$
\begin{gathered}
\eta\left(k+1, r^{\prime}, \boldsymbol{s} \boldsymbol{w}^{\boldsymbol{c}}\right) \leq \eta\left(k, r^{\prime}, \boldsymbol{s} \boldsymbol{w}^{\boldsymbol{c}}\right) \quad \Leftrightarrow \quad \eta\left(\{k, k+1\}, r^{\prime \prime}, \boldsymbol{s} \boldsymbol{w}^{\boldsymbol{c}}\right)=0 \quad \forall r^{\prime \prime} \neq r^{\prime} \\
k, k+1 \in \boldsymbol{B}, \quad r^{\prime}, r^{\prime \prime}=1, \ldots, N_{R}
\end{gathered}
$$

in which bus $k$ is assumed to be closer to source $r$ than bus $k+1$. Eqs. (7)-(9) hold for any set of trees representing a viable configuration c. The next restriction defines the upper and lower limits for bus voltages:

$V_{k}^{\min } \leq V_{k} \leq V_{k}^{\max }, \quad \forall k=1, \ldots, N_{B}$

Known also as thermic limit, line ampacity must also be obeyed:

$\left|I_{i}\right| \leq I_{i}^{\max }, \quad \forall i \in L$

Finally, none of the $N_{R}$ sources is admitted to be overloaded. Power demand of the busses fed by source $r$ must not exceed its power availability $S_{r}^{\max }$ when considering lines losses $\left(L L^{r}\right)$ associated with each $r$ th tree:

$\left|\sum_{k=1}^{N_{B}} \eta\left(k, r, \boldsymbol{s} \boldsymbol{w}^{c}\right) \cdot\left(P_{k}+j Q_{k}\right)+L L^{r}\right|<S_{r}^{\max }, \quad \forall r=1, \ldots, N_{R}$

In Eq. (11), line losses can be computed through an expression very similar to Eq. (1) in which $\operatorname{Re}\left(Z_{i}\right)$ shall be replaced by $Z_{i}$. Added to topological constraint, equality constraints include loadflow equations given by Kirchhoff voltage and current laws. They are generically represented here as:

$\Phi\left(\mathbf{s} \boldsymbol{w}^{c}\right)=0$ 


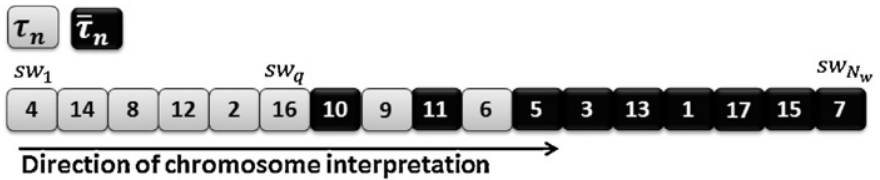

Fig. 1. Chromosome interpretation scheme.

\section{Approach outline}

Although evolutionary search techniques do not necessarily guarantee global optima, they are well suited to problems such as DFR. Because they tend to avoid entrapments offered by local optima during the search, global optima are more likely to be reached and therefore better performance can be attained than employing pure heuristics or non-evolutionary meta-heuristics. In this work, reconfiguration optimization is performed through an evolutionary algorithm based on the NSGA-II [10]. Some adaptions are suggested to improve the overall performance of the application as well as its suitability to the addressed problem. Most of the reviewed proposals seen in Section 2 have specific procedures to validate configurations found along the optimization process. Even supposing that evolutionary search process starts up from a feasible population, unfeasible offspring may be produced by using common genetic operators. In those cases, it is necessary to implement one of the following typical options: (1) chromosome repairing; (2) genetic operator reapplying; or (3) chromosome discard. These procedures not only require additional computation but they also distort the paradigm of natural evolution [7]. If the implemented algorithm ensures feasibility for every single solution proper to DFR problem, thus running time required by evolutionbased approaches can be considerably reduced. Ideally, an encoding algorithm must perform a straight translation between any possible configuration to its equivalent chromosome representation. Reversely, a decoding algorithm is also needed for the interpretation of the chromosome.

\subsection{Codification and individual interpretation}

Chromosome coding scheme is an important issue to the success of an evolutionary algorithm application. The codification may support good convergence for EA supposing that it has some desired properties. As already known, an efficient codification ensures coverage and bias, demands minor complex adaptions on stochastic operators and causes no harm to population diversity [29]. Any extra computation for configuration validation or repairing must be avoided as much as possible. Here, any possible network configuration is represented through an ordered integer array as seen in Fig. 1. Assuming that each switchable line belonging to the set $\boldsymbol{\Omega}$ is identified by a unique integer, gene alleles can assume any of those values. Thus, there is no concern about validity of the ordered chromosome since it is the interpretation or decoding procedure who maps any chromosome content into a feasible configuration. This interpretation procedure is sequential as in [8] but this proposal does not demand preprocessing as in $[8,36,37]$. To decode chromosome, a simple algorithm to be described in the next section is carried out. The represented configuration $\mathfrak{F}$ is retrieved by means of a unidirectional sweep made over genes, conventionally defined in the implementation (from left to right, for example). During this interpretation, sets $\boldsymbol{\tau}$ and $\overline{\boldsymbol{\tau}}$ are naturally obtained which is useful information to be applied in a kind of mutation explained in Section 4.3. This simplicity about the decoding procedure is due to the straight correspondence between graph representation and coding scheme. It also leads to efficiently computation, although chromosome length is proportional to the number of switchable lines. As we previously know the desired cardinalities of $\boldsymbol{\tau}$ and $\overline{\boldsymbol{\tau}}$, sweeping all the chromosome genes is not always needed once the number of lines to be kept closed have already been found. On average, $\|\boldsymbol{\tau}\|+\|\overline{\boldsymbol{\tau}}\| / 2$ positions will be swept. Thus, decoding time varies from chromosome to chromosome. It is noteworthy to mention that repairing, which weakens locality and heritability properties, is needless in our approach since unfeasible solutions are circumvented by the way genes are decoded. Neither loop detection procedure is demanded. Besides that, complementary data structure is not used and hence this approach does not require considerable memory space as in [40]. Being suited for full-connected or sparse graphs, our decoding procedure does not impose any restriction to chromosome values and all possible sequence combination can always be mapped into a feasible configuration. Distinctly from Carreno [29], in our coding scheme genetic operators are not susceptible to gene positioning in order to ensure offspring feasibility. Also, our scheme does not employ matrices as found in [8] and it performs any operation directly on the graph which represents the network. The correspondence property [9,24] is not hold for the proposed representation since it is possible to a given configuration be described by two or more distinct vectors. However, the same occurred for other techniques: main chain [38], edge set [9], and matroids [28]. The mentioned drawback is demonstrated to be harmless to the optimization due to the results attained by experimental simulations, as seen in Section 5 .

\subsection{Labeling constructive algorithm}

The labeling constructive algorithm (LCA) is a constructive-like branch encoding strategy oriented through graph analysis which is employed here to create any configuration. Let $G(\boldsymbol{B}, \boldsymbol{L})$ be a connected and undirected graph on $\boldsymbol{B}$ where $\boldsymbol{B}=\left\{1,2, \ldots, N_{B}\right\}$ is the set of nodes (busses) and $\boldsymbol{L}$ is the finite set of edges (lines). Better said, the set of unordered pairs represents pairs $\{k, l\} \in \boldsymbol{B} \times \boldsymbol{B}$ of distinct nodes connected $(k \neq l)$ indeed. We developed a simple and straight algorithm capable of building trees from any graph $G$. In case of multiple sources $G$, must represent a forest instead of a single tree, since distribution systems operate as a group of radial feeders [1]. Thus, let $\mathfrak{F}\left(G ; N_{B}\right)$ denote a group of all set of viable $N_{R}$ trees with $N_{B}$ busses obtained from $G$ containing no cycles.

The key of the LCA is the label assignment made to each graph node. Those labels shall assume positive, negative or zero values indicating an energized node, non-energized node or nonassignment, respectively. The edges are identified with unique positive numbers. In our approach, there is no need to know fundamental loops as well as the edges which constitute them. Unlike Kruskal [41] and Prim [42,43] algorithms, the LCA can easily create a forest and it is generic enough to handle graphs under any level of construction. In Algorithm 1, the main steps of the LCA are given. Being proper to reconfiguration or restoration cases, single source or multiple sources, its implementation is rather straight and no major modifications must be done on genetic operators, as we will see in Section 4.3. On edge inclusion analysis mentioned in line 12 of Algorithm 1, two basic rules must be adhered:

(I) edge $e$ cannot be included if (i) end nodes have positive labels, or (ii) they have identical negative values;

(II) edge $e$ is allowed to be included whenever (i) one of the two end nodes is zero labeled, or (ii) they are labeled with distinct negative values, or (iii) one label is positive while the other is not positive. 


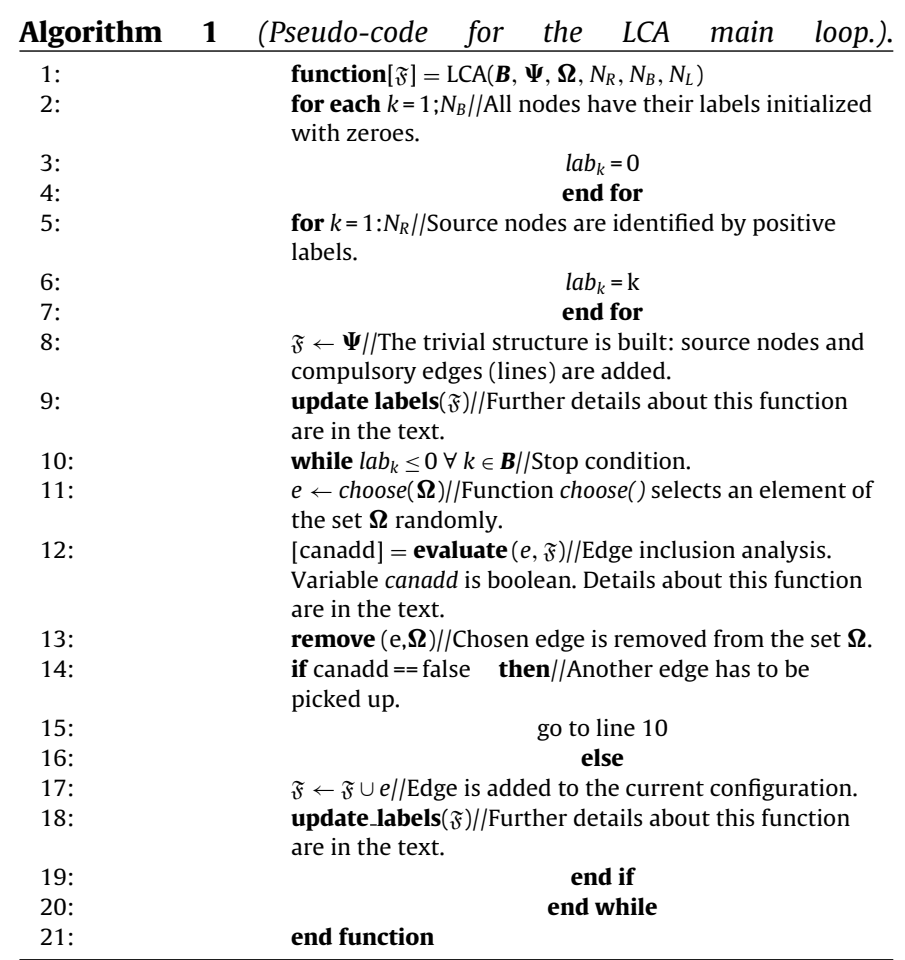

Finally, the update procedure called in lines 9 and 18 of Algorithm 1 is implemented as follows:

(i) if end nodes are labeled with zeroes, they will assume the next available negative value;

(ii) if one end node is zero, this node will receive the label value from the other end node;

(iii) and if both end nodes are labeled with distinct negative values, the lowest value will be copied to the other end node and will be disseminated across every node connected to it replacing former label values.

To better understand, a fictitious network example is given in Fig. 2(a)-(i) where the chromosome presented in Fig. 1 is gradually built up. For this example, we suppose that edges were randomly chosen and this sequence is equal to the one shown in Fig. 1. Three sources are admitted for this network. A legend provided at the top of Fig. 2 makes clear the meaning of the graphic symbols. The meaning of the numbers inside each node was already explained. Dashed lines represent edges not interpreted yet. Both continuous and dotted-cross lines are the edges already interpreted by the LCA. The configuration showed in Fig. 2(i) has 3 sources, 8 load busses, and 17 lines. Admitting that every line has a switch, it is trivial to find that $\|\boldsymbol{\tau}\|=8$ and $\|\overline{\boldsymbol{\tau}}\|=9$ and using Eqs. (7a) and (7b) which are the same for any feasible configuration derived from it.

\subsection{Stochastic operators}

Based on our codification scheme, it is possible to adopt stochastic operators as they were originally conceived [44]. In spite of that, other mutation operators were proposed in order to survey potential improvements on overall search performance of the evolutionary algorithm. Thus, we employed PMX crossover [29], eight mutation types explained later in this section and local elitism as a stochastic operators suite. Performing combination between two chromosomes by means of the PMX crossover, two new individuals are generated as demonstrated in Fig. 3. In that figure, the first parent is the reference chromosome and two points are fixed to delimit the segments with different background colors to be interchanged between parents. In the offsprings, highlighted genes indicate allele reallocation provided by PMX. Since codification makes the chromosomes ordered, PMX is a proper recombination operator. Generally, mutation is responsible for the occasional random alteration of genes. In DFR problem, mutation is equivalent to an exchange (swap) operation that closes an open switch and opens a closed switch in the loop formed so that radiality and connectivity are still retained. The parameter $m r$ controls the rate new solutions are explored in the DFR landscape. If this rate is high, offspring lose resemblance to their parents and the algorithm could not learn from the past exposing an erratic behavior. Ideally, mutation should promote positive changes on individual leading search to its neighborhood. A neighboring solution is assumed here as being a feasible configuration that differs by a pair of open-close switching maneuvers.

Considering mutation effects over chromosome, we proposed some permutations that cause similarly behavior through minor changes on chromosome content to preserve heritage in relation to the general characteristics of the prime configuration. Three basic mutation schemes are shown in Fig. 4(a) in which the prime individual is the reference configuration already presented by Figs. 1 and 2(i). That configuration is also considered as a reference to the three elaborated mutation schemes shown in Fig. 4(b). Basic mutation types involve simple swapping schemes. In mutation type 1 (MT1), any pair of genes can be interchanged. Once applied, there is still a probability that the chromosome content continues to represent the same configuration. Therefore, this alternative is somewhat inefficient. See Fig. 4(a) for clarity. Using MT1, it is more likely to scramble the chromosome content than providing a different configuration effectively.

Through mutation type 2 (MT2), any gene located at the first $\|\boldsymbol{\tau}\|$ positions of the chromosome is assured to be swapped by other located in the last $\|\overline{\boldsymbol{\tau}}\|$ positions. The MT2 increases the probability of the mutation actually performs any modification in the original configuration as shown in Fig. 4(a). Mutation type 3 (MT3) needs chromosome decoding to identify the set $\overline{\boldsymbol{\tau}}$. One element of this set is picked up at random to be swapped out with an element from its complementary set $\boldsymbol{\tau}$ situated preferably in an upright position. In case of MT3, chromosomes to be mutated must be decoded first (see Fig. 4(a)). For mutation types 4 and 5, the parameter $\delta$, that controls a fixed percentage of $\|\overline{\boldsymbol{\tau}}\|$ to be changed, is included to allow sharper modifications. MT4 and MT5 employ respectively a uniform and Gaussian probability distribution functions to define how many swaps will occur whenever a new mutation is about to happen. Mutations MT4 and MT5 can be viewed schematically in Fig. 4(b). The next mutation type considered (MT6) is similar to MT4 but it is more intense because it takes a percentage of genes from the whole chromosome, instead of $\|\overline{\boldsymbol{\tau}}\|$. At last, in mutation type 7 , one element of the set $\boldsymbol{\tau}$ is moved to the rightmost position of the chromosome while mutation type 8 moves one element of the set $\|\boldsymbol{\tau}\|$ to the leftmost position. For both mutation types, the remaining genes must be properly shifted to the opposite side, seen in Fig. 4(b). As pointed out at the beginning of this section, we implemented local elitism by selecting the best configurations among parents, offspring obtained after crossover and after mutation.

\subsection{Fitness evaluation}

A load flow method is the basis for the configuration evaluation. After its convergence, it is possible to determine voltages, currents and power losses which correspond to the power system state. Thus, the first objective detailed in Section 3 is calculated through a backward-forward sweep power flow method (BFSM). But other methods could be equally applied such as the compensation-based for weakly meshed networks [45]. In this work, we employed three 

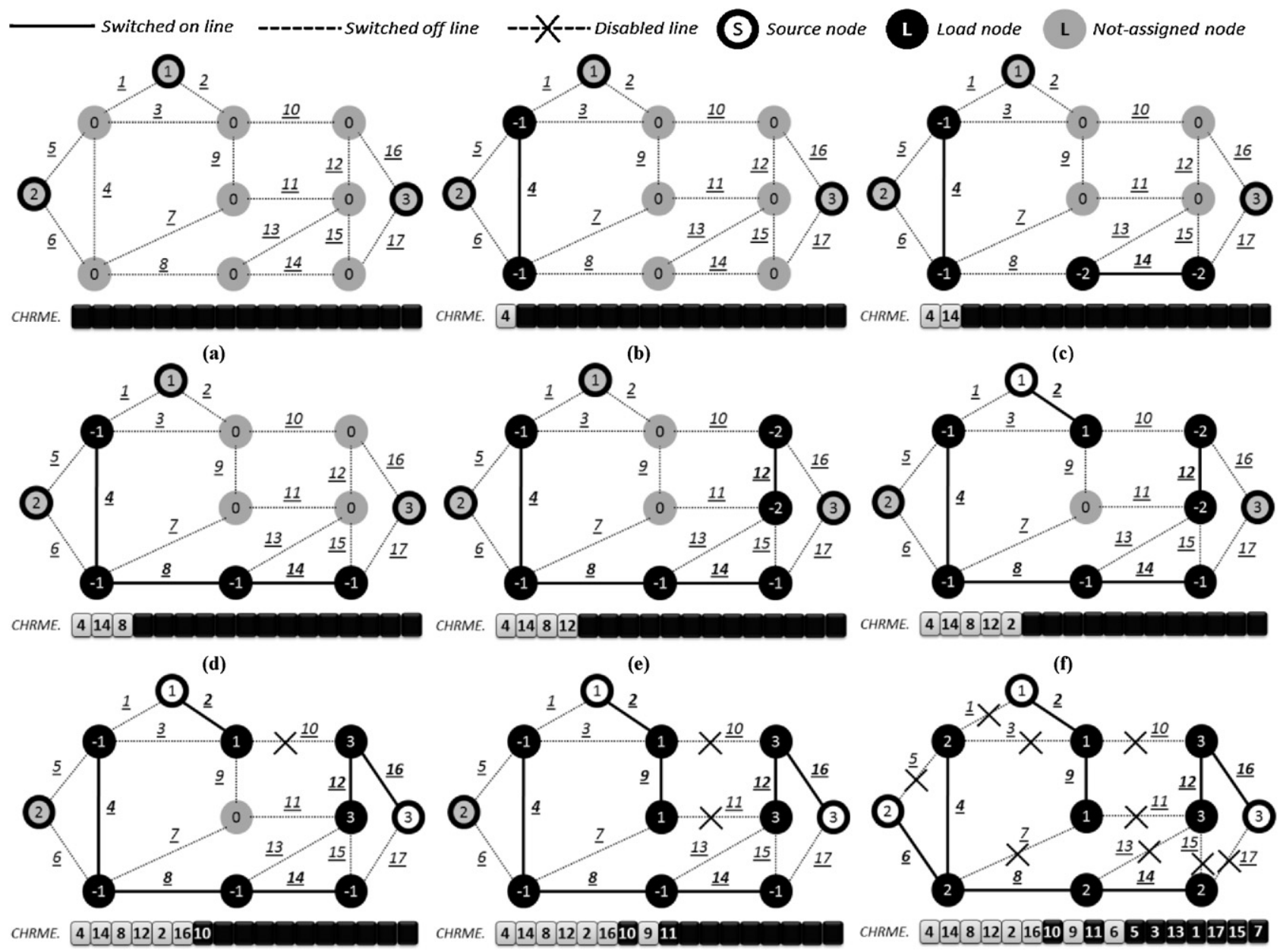

(g)

(h)

(i)

Fig. 2. Obtaining $N_{R}$ trees from a graph $G(\boldsymbol{B}, \boldsymbol{L})$ through LCA steps according to the sequence $\{4,14,8,12,2,16,10,9,11,6,5,3,13,1,17,15,7\}$ randomly generated.

variations of BFSM: (1) power aggregation with simplified formulation based on distflow equations [14] (BFSM-PA\#1) which neglects line losses [31]; (2) power aggregation considering line losses (BFSM-PA\#2) based on Su's work [3]; and (3) current aggregation (BFSM-CA) [46]. We implemented those BFSM versions to compare results with other works. The convergence tolerance to the three BFSM was adjusted for $1 \times 10^{-6}$ taking three iterations on average to converge. A basic scheme of a genetic algorithm tailored for the DFR problem can be viewed in [25]. Here, we employed the NSGA-II to deal with multiple criteria. No further details are given once similar scopes for it were presented in [32,37]. As seen in Section 3, DFR problem includes several electrical and topological

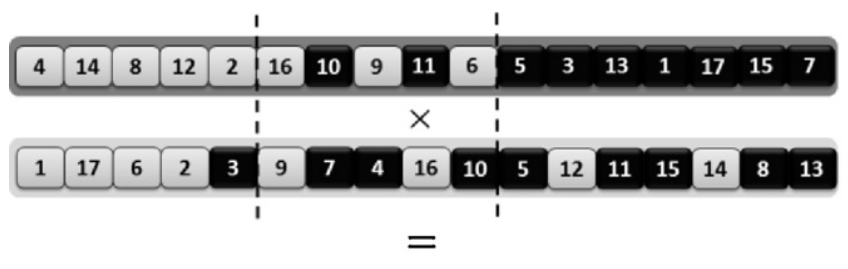

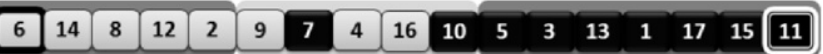

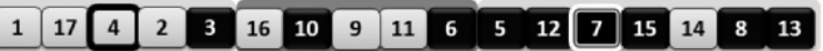

Fig. 3. A PMX crossover example. constraints. Ensuring that topologically feasible individuals are created during population evolution in EA, we must concern only with electrical constraints during dominance analysis. Here, dominance analysis is done as in [10]: (a) non-violated solution is preferred over violated ones, (b) the least violated solution is chosen when two violated are compared, and (c) two feasible solutions are compared according to Pareto dominance. Distribution systems have been adopting an increasingly number of distributed generation (DG) units. In this sense, local loops with power bidirectionally flowing in those systems have created a new challenge for the operation personnel. Few adaptions in our approach could make DG easily tackled once those loops can be treated by the LCA as single nodes, each represented along with the equivalent net power. Thus, optimization configuration could be done as usual.

\section{Experimental results}

In this work, five distribution systems already tested by published papers were considered. General details about the systems are given in Table 1 . Four systems are schematically illustrated in Refs. [1,13,31], and [3], respectively. The 136-bus system is illustrated in Fig. 5. The ampacity values for lines were not defined for Systems \#3, \#4 and \#5. So, those values were estimated in order to allow CLI calculation. In System \#3, a value of $1.2 \mathrm{kA}$ was assumed for all lines since more than $90 \%$ of the topologically viable configurations did not violate the current indexes $(C L I<1.0)$. For System \#4, 


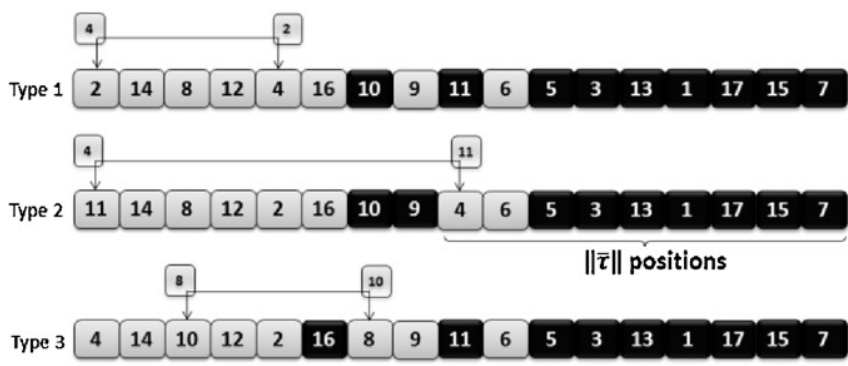

(a)

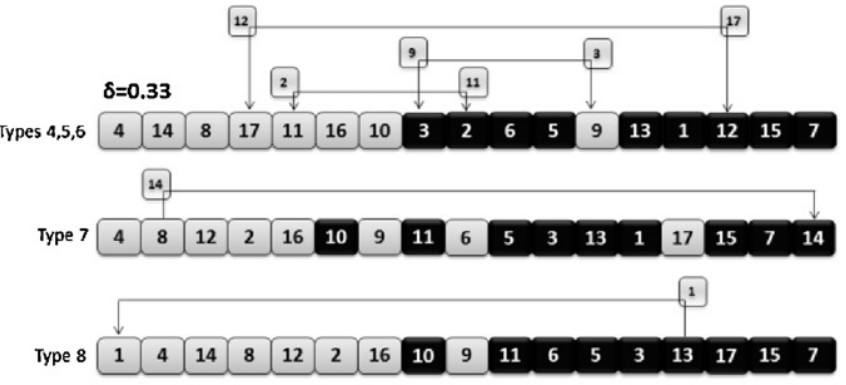

(b)

Fig. 4. (a) Basic mutation types 1-3 and (b) elaborated mutation types 4-8.

Table 1

Topology details, electrical specifications, and dimension of the space search for the five tested systems.

\begin{tabular}{|c|c|c|c|c|c|c|c|c|c|c|c|}
\hline System & Refs. & $N_{R}$ & $N_{B}$ & $N_{L}$ & $N_{W}$ & $N L$ & $S_{\text {base }}$ (MVA) & $V_{\text {base }}(\mathrm{kV})$ & $\begin{array}{l}\text { Number of } \\
\text { possibilities }\end{array}$ & Combination $\left(\begin{array}{c}N_{W S} \\
N L\end{array}\right)$ & $\begin{array}{l}\text { Topologically } \\
\text { feasible } \\
\text { configurations }\end{array}$ \\
\hline 1 & {$[1,14]$} & 1 & 33 & 37 & 37 & 5 & 10 & 12.66 & $2^{37}$ & 376,992 & $50,751^{c}$ \\
\hline 2 & {$[27,31,25]$} & 1 & 70 & 74 & 74 & 5 & 10 & 12.66 & $2^{74 b}$ & $15,020,334$ & 376,028 \\
\hline 3 & {$[1,47,36,13,17,48]$} & 3 & 16 & 16 & 16 & 3 & $100^{\mathrm{a}}$ & 12.66 & $2^{16}$ & 560 & 190 \\
\hline 4 & [3] & 11 & 94 & 96 & 96 & 13 & 100 & 11.4 & $2^{96}$ & $4.036 \times 10^{15}$ & $3.5196 \times 10^{11}$ \\
\hline 5 & [29] & 1 & 136 & 156 & 156 & 21 & 100 & 13.8 & $2^{156}$ & $5.435 \times 10^{25}$ & $2.2686 \times 10^{18}$ \\
\hline
\end{tabular}

a This value is not provided in [1].

b All lines are considered to have a switch as stated in the original paper [31].

c Also reported in Morton's work [49].

a mean value of $655 \mathrm{~A}$ was defined to represent admissible currents in ACSR 477 and $500 \mathrm{KCM}$ conductors.

Experiments were performed for BFSM-PA \#1 and \#2, and BFSM-CA. In Table 1, information about search space size for all systems is also provided. The number of radial configurations was obtained by an exhaustive search such that described in [49]. The number of combination was deduced from $N_{W S} ! /\left[\left(N_{W S}-N L\right) ! \cdot N L !\right]$ at Table 1 . A computational platform with 2-core $1.66 \mathrm{GHz}$ processor and 2GB RAM memory was used in all experiments described hereinafter. Program codes were developed in MatLab ${ }^{\mathrm{TM}}$ language version 7.8.0.

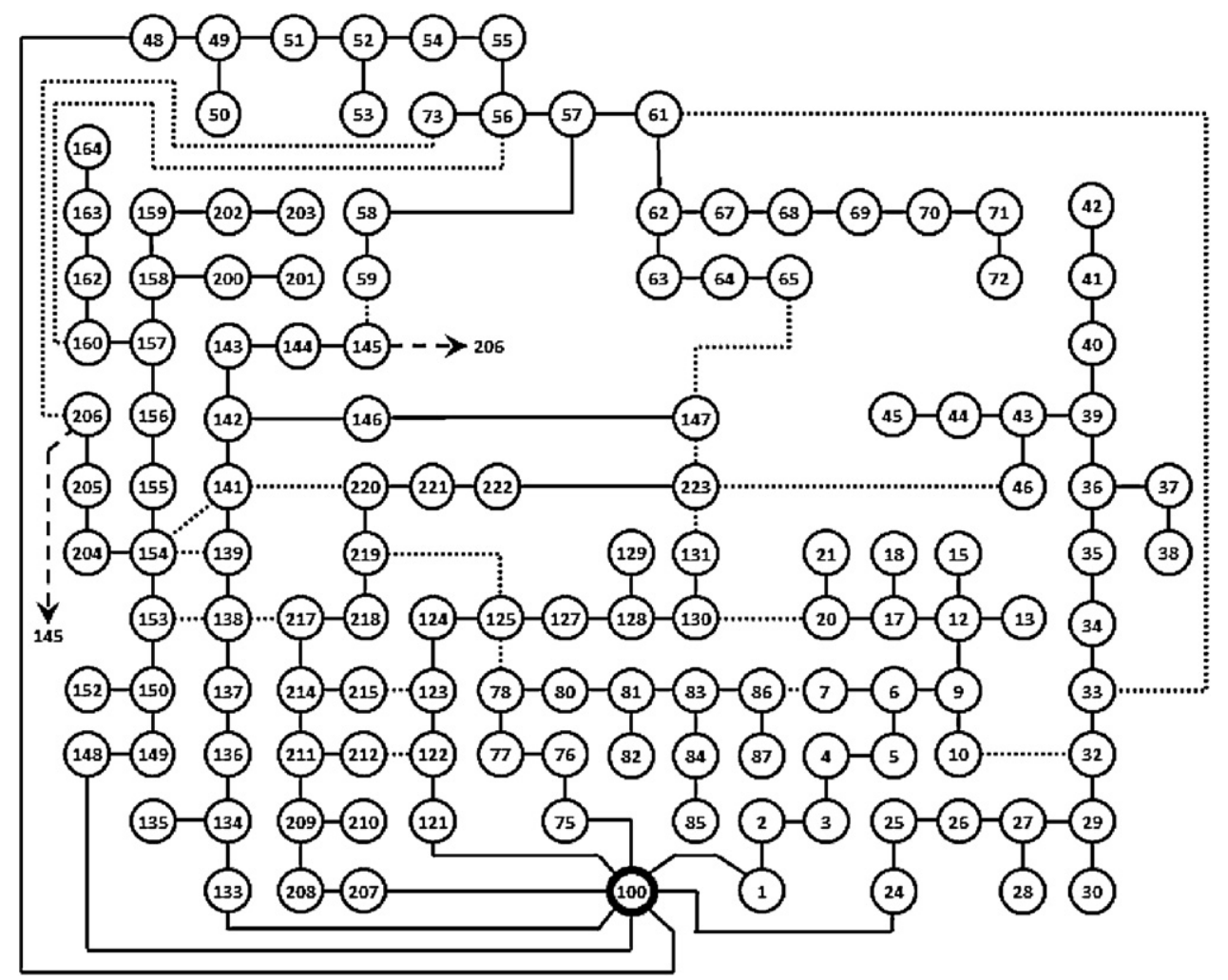

Fig. 5. A 136-bus Brazilian distribution system described in [29]. 
Table 2

Mono-objective optimization performed for System \#1 using two BFSMs.

\begin{tabular}{|c|c|c|c|c|c|c|}
\hline \multirow[t]{2}{*}{ Simulation case } & \multicolumn{3}{|l|}{ BFSM-PA\#1 } & \multicolumn{3}{|l|}{ BFSM-PA\#2 } \\
\hline & $\begin{array}{l}\text { Best solution } \\
\text { achieved }\end{array}$ & $\begin{array}{l}\text { Average CPU time } \\
\text { (s) }\end{array}$ & $\begin{array}{l}\text { Convergence rate } \\
\text { for } 33 \text { trials (\%) }\end{array}$ & $\begin{array}{l}\text { Best solution } \\
\text { achieved }\end{array}$ & $\begin{array}{l}\text { Average CPU time } \\
\text { (s) }\end{array}$ & $\begin{array}{l}\text { Convergence rate } \\
\text { for } 33 \text { trials (\%) }\end{array}$ \\
\hline $\begin{array}{c}\text { As originally } \\
\text { proposed }\end{array}$ & s7-s9-s14-s32-s37 & 2.13 & 100 & s7-s9-s14-s32-s37 & 2.95 & 100 \\
\hline $\begin{array}{r}\text { Different initial } \\
\text { configuration }\end{array}$ & s7-s9-s14-s32-s37 & 2.18 & 100 & s7-s9-s14-s32-s37 & 2.93 & 100 \\
\hline $\begin{array}{r}\text { Load modified at } \\
\text { buses } 9 \text { and } 13\end{array}$ & s9-s14-s28-s32-s33 & 2.19 & 100 & s9-s14-s28-s32-s33 & 2.99 & 100 \\
\hline
\end{tabular}

Table 3

Mono-objective optimization performed for System \#2 using BFSM-PA\#1.

\begin{tabular}{|c|c|c|c|}
\hline Method & Best solutions achieved ${ }^{a}$ & Power losses (kW) & CPU time $(\mathrm{s})$ \\
\hline $\begin{array}{l}\text { Exhaustive } \\
\text { search }\end{array}$ & $\begin{array}{l}\text { s13-s21-s56-s62-s70, } \\
\text { s13-s21-s57-s62-s70, } \\
\text { s13-s21-s58-s62-s70, } \\
\text { s13-s21-s59-s62-s70 }\end{array}$ & 94.026 & - \\
\hline Proposed & $\begin{array}{l}\text { s13-s21-s56-s62-s70, } \\
\text { s13-s21-s57-s62-s70, } \\
\text { s13-s21-s58-s62-s70, } \\
\text { s13-s21-s59-s62-s70 }\end{array}$ & 94.026 & 9.4 \\
\hline Huang [31] & s13-s59-s70-s71-s74 & 113.406 & Not indicated \\
\hline Savier [50] & s15-s57-s64-s70-s71 & 99.59 & 1.8 \\
\hline Swarnkar [25] & s15-s57-s62-s70-s71 & 99.59 & 3.3 \\
\hline
\end{tabular}

a Switches ID's are took as referenced in [31].

\subsection{Mono-objective optimization}

A mono-objective analysis of the DFR problem aimed at the minimization of real power losses was carried out using a classical genetic algorithm (GA) [44]. The global optimum of System \#1, obtained by exhaustive evaluation reported in [48], was successfully achieved by our approach for the three cases evaluated by Gomes [48]: original system, distinct initial configuration, and load modification at buses 9 and 13. The correspondent results are presented in Table 2. Initial configuration was included in the GA first population for cases 1 and 2 while remainder chromosomes were randomly generated. It is noteworthy that the optima were attained in all cases regardless the inclusion of the initial configuration in the GA first population. The operators MT3 and MT7 achieved the best performance. Our approach has also succeeded in finding the best configurations for System \#2. Distinctly from other references, we found four configurations which coincided with the solutions reached by an exhaustive search. Results are summarized in Table 3. Tests on System \#3 using BFSM-PA\#2 have outlined consistency in converging to the same solution found by Zhu [1]. It took $0.08 \mathrm{~s}$ in average to achieve the best network configuration with open switches s6, s9 and s11 leading to losses of $466.1 \mathrm{~kW}$. About 30 executions were tried considering 200 evaluations for a population size of 20 chromosomes. Using BFSM-PA\#1, we also found the same optimum with power losses of $449.67 \mathrm{~kW}$. System \#4 was also optimized with the same formulation used by Su [3] (BFSM-PA\#2). Same solution reported in that work was found after 112.8 s (s7-s13-s34-s39-s41s55-s62-s72-s83-s86-s89-s90-s92), which is the configuration of $469.88 \mathrm{~kW}$.

Table 4

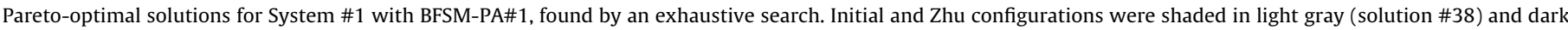
gray (solution \#4), respectively.

\begin{tabular}{|c|c|c|c|c|c|c|c|c|c|c|c|c|c|c|c|c|c|c|c|}
\hline \multirow[t]{2}{*}{ Solution } & \multicolumn{5}{|c|}{$\begin{array}{l}\text { Variable domain } \\
\text { (opened switches) }\end{array}$} & \multicolumn{4}{|c|}{ Objective domain } & \multirow[t]{2}{*}{ Solution } & \multicolumn{5}{|c|}{$\begin{array}{l}\text { Variable domain } \\
\text { (opened switches) }\end{array}$} & \multicolumn{4}{|c|}{ Objective domain } \\
\hline & $\mathrm{S} 1$ & S2 & S3 & $\mathrm{S} 4$ & S5 & $\mathrm{PL}(\mathrm{kW})$ & VDI & CLI & NS & & $\mathrm{S} 1$ & $\mathrm{~S} 2$ & S3 & S4 & S5 & $\mathrm{PL}(\mathrm{kW})$ & VDI & CLI & NS \\
\hline 1 & 7 & 9 & 1427 & 37 & & 136.8573 & 0.0326 & 0.5480 & 8 & 20 & 7 & 13 & 27 & 35 & 37 & 146.2070 & 0.0369 & 0.5575 & 6 \\
\hline 2 & 7 & 9 & 14 & 28 & 32 & 132.1250 & 0.0318 & 0.5511 & 10 & 21 & 7 & 13 & 28 & 35 & 37 & 143.1198 & 0.0357 & 0.5591 & 6 \\
\hline 3 & 7 & 9 & 14 & 28 & 37 & 133.7701 & 0.0314 & 0.5496 & 8 & 22 & 8 & 33 & 34 & 36 & 37 & 144.1015 & 0.0368 & 0.6519 & 2 \\
\hline 4 & 7 & 9 & 14 & 32 & 33 & 131.8484 & 0.0339 & 0.6218 & 8 & 23 & 9 & 14 & 27 & 34 & 37 & 140.4228 & 0.0320 & 0.5731 & 6 \\
\hline 5 & 7 & 9 & 14 & 33 & 37 & 134.0667 & 0.0338 & 0.6251 & 6 & 24 & 9 & 14 & 28 & 32 & 34 & 136.3446 & 0.0312 & 0.5793 & 8 \\
\hline 6 & 7 & 10 & 14 & 27 & 32 & 135.6836 & 0.0332 & 0.5482 & 10 & 25 & 9 & 14 & 28 & 34 & 37 & 138.0678 & 0.0311 & 0.5748 & 6 \\
\hline 7 & 7 & 10 & 14 & 27 & 37 & 137.3173 & 0.0328 & 0.5468 & 8 & 26 & 9 & 17 & 28 & 34 & 36 & 141.1753 & 0.0324 & 0.5721 & 6 \\
\hline 8 & 7 & 10 & 14 & 28 & 32 & 132.7685 & 0.0321 & 0.5498 & 10 & 27 & 9 & 27 & 34 & 36 & 37 & 140.5511 & 0.0336 & 0.5730 & 4 \\
\hline 9 & 7 & 10 & 14 & 28 & 37 & 134.2301 & 0.0316 & 0.5484 & 8 & 28 & 9 & 28 & 34 & 36 & 37 & 137.8256 & 0.0326 & 0.5746 & 4 \\
\hline 10 & 7 & 10 & 14 & 32 & 33 & 132.4920 & 0.0342 & 0.6205 & 8 & 29 & 9 & 33 & 34 & 36 & 37 & 144.5005 & 0.0364 & 0.6503 & 2 \\
\hline 11 & 7 & 10 & 14 & 33 & 37 & 134.5267 & 0.0340 & 0.6238 & 6 & 30 & 10 & 27 & 34 & 36 & 37 & 140.0848 & 0.0330 & 0.5743 & 4 \\
\hline 12 & 7 & 10 & 27 & 36 & 37 & 139.4704 & 0.0341 & 0.5593 & 6 & 31 & 10 & 28 & 32 & 34 & 36 & 135.7674 & 0.0326 & 0.5806 & 6 \\
\hline 13 & 7 & 10 & 28 & 36 & 37 & 136.3832 & 0.0329 & 0.5609 & 6 & 32 & 10 & 28 & 34 & 36 & 37 & 137.4405 & 0.0320 & 0.5759 & 4 \\
\hline 14 & 7 & 10 & 33 & 36 & 37 & 136.6798 & 0.0353 & 0.6363 & 4 & 33 & 10 & 33 & 34 & 36 & 37 & 145.4609 & 0.0363 & 0.6516 & 2 \\
\hline 15 & 7 & 11 & 27 & 36 & 37 & 138.9756 & 0.0337 & 0.5604 & 6 & 34 & 11 & 28 & 32 & 34 & 36 & 135.6151 & 0.0321 & 0.5840 & 6 \\
\hline 16 & 7 & 11 & 28 & 36 & 37 & 135.8884 & 0.0325 & 0.5620 & 6 & 35 & 11 & 28 & 34 & 36 & 37 & 137.5742 & 0.0317 & 0.5794 & 4 \\
\hline 17 & 7 & 11 & 33 & 36 & 37 & 136.1850 & 0.0349 & 0.6374 & 4 & 36 & 27 & 34 & 35 & 36 & 37 & 165.2175 & 0.0412 & 0.6431 & 2 \\
\hline 18 & 7 & 12 & 27 & 35 & 37 & 146.8668 & 0.0372 & 0.5560 & 6 & 37 & 28 & 34 & 35 & 36 & 37 & 163.3912 & 0.0406 & 0.6449 & 2 \\
\hline 19 & 7 & 12 & 28 & 35 & 37 & 143.7797 & 0.0360 & 0.5576 & 6 & 38 & 33 & 34 & 35 & 36 & 37 & 186.0441 & 0.0496 & 0.7233 & 0 \\
\hline
\end{tabular}


Table 5

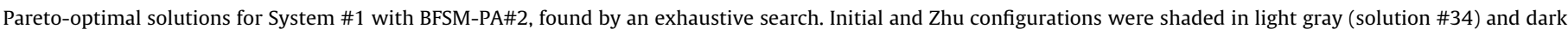
gray (solution \#3), respectively.

\begin{tabular}{|c|c|c|c|c|c|c|c|c|c|c|c|c|c|c|c|c|c|c|c|}
\hline \multirow[t]{2}{*}{ Solution } & \multicolumn{5}{|c|}{$\begin{array}{l}\text { Variable domain } \\
\text { (opened switches) }\end{array}$} & \multicolumn{4}{|c|}{ Objective domain } & \multirow[t]{2}{*}{ Solution } & \multicolumn{5}{|c|}{$\begin{array}{l}\text { Variable domain } \\
\text { (opened switches) }\end{array}$} & \multicolumn{4}{|c|}{ Objective domain } \\
\hline & S1 & S2 & S3 & S4 & S5 & $\mathrm{PL}(\mathrm{kW})$ & VDI & CLI & NS & & $\mathrm{S} 1$ & $\mathrm{~S} 2$ & S3 & S4 & S5 & $\mathrm{PL}(\mathrm{kW})$ & VDI & CLI & NS \\
\hline 1 & 7 & 9 & 14 & 28 & 32 & 139.9823 & 0.0326 & 0.5650 & 10 & 18 & 8 & 33 & 34 & 36 & 37 & 153.4974 & 0.0378 & 0.6707 & 2 \\
\hline 2 & 7 & 9 & 14 & 28 & 37 & 141.9213 & 0.0322 & 0.5636 & 8 & 19 & 9 & 14 & 27 & 34 & 37 & 149.4261 & 0.0327 & 0.5878 & 6 \\
\hline 3 & 7 & 9 & 14 & 32 & 33 & 139.5542 & 0.0348 & 0.6381 & 8 & 20 & 9 & 14 & 28 & 32 & 34 & 144.5838 & 0.0319 & 0.5936 & 8 \\
\hline 4 & 7 & 9 & 14 & 33 & 37 & 142.1687 & 0.0347 & 0.6420 & 6 & 21 & 9 & 14 & 28 & 34 & 37 & 146.6722 & 0.0319 & 0.5891 & 6 \\
\hline 5 & 7 & 10 & 14 & 27 & 37 & 145.9686 & 0.0336 & 0.5613 & 8 & 22 & 9 & 17 & 28 & 34 & 36 & 150.4038 & 0.0332 & 0.5872 & 6 \\
\hline 6 & 7 & 10 & 14 & 28 & 32 & 140.7099 & 0.0329 & 0.5638 & 10 & 23 & 9 & 27 & 34 & 36 & 37 & 149.5296 & 0.0345 & 0.5882 & 4 \\
\hline 7 & 7 & 10 & 14 & 28 & 37 & 142.4342 & 0.0324 & 0.5624 & 8 & 24 & 9 & 28 & 34 & 36 & 37 & 146.3735 & 0.0334 & 0.5894 & 4 \\
\hline 8 & 7 & 10 & 14 & 32 & 33 & 140.2818 & 0.0351 & 0.6370 & 8 & 25 & 9 & 33 & 34 & 36 & 37 & 153.9966 & 0.0374 & 0.6690 & 2 \\
\hline 9 & 7 & 10 & 14 & 33 & 37 & 142.6815 & 0.0349 & 0.6408 & 6 & 26 & 10 & 27 & 34 & 36 & 37 & 148.9915 & 0.0338 & 0.5893 & 4 \\
\hline 10 & 7 & 10 & 27 & 36 & 37 & 148.3988 & 0.0350 & 0.5744 & 6 & 27 & 10 & 28 & 32 & 34 & 36 & 143.9339 & 0.0334 & 0.5954 & 6 \\
\hline 11 & 7 & 10 & 28 & 36 & 37 & 144.8643 & 0.0337 & 0.5756 & 6 & 28 & 10 & 28 & 34 & 36 & 37 & 145.9220 & 0.0328 & 0.5906 & 4 \\
\hline 12 & 7 & 10 & 33 & 36 & 37 & 145.1117 & 0.0362 & 0.6540 & 4 & 29 & 10 & 33 & 34 & 36 & 37 & 155.1353 & 0.0374 & 0.6704 & 2 \\
\hline 13 & 7 & 11 & 27 & 36 & 37 & 147.8276 & 0.0346 & 0.5754 & 6 & 30 & 11 & 28 & 32 & 34 & 36 & 143.7160 & 0.0329 & 0.5986 & 6 \\
\hline 14 & 7 & 11 & 28 & 36 & 37 & 144.2932 & 0.0333 & 0.5766 & 6 & 31 & 11 & 28 & 34 & 36 & 37 & 146.0456 & 0.0325 & 0.5940 & 4 \\
\hline 15 & 7 & 11 & 33 & 36 & 37 & 144.5405 & 0.0358 & 0.6550 & 4 & 32 & 27 & 34 & 35 & 36 & 37 & 177.2866 & 0.0424 & 0.6625 & 2 \\
\hline 16 & 7 & 12 & 27 & 35 & 37 & 156.7469 & 0.0382 & 0.5722 & 6 & 33 & 28 & 34 & 35 & 36 & 37 & 175.1384 & 0.0418 & 0.6640 & 2 \\
\hline 17 & 7 & 12 & 28 & 35 & 37 & 153.2123 & 0.0370 & 0.5734 & 6 & 34 & 33 & 34 & 35 & 36 & 37 & 202.6861 & 0.0515 & 0.7492 & 0 \\
\hline
\end{tabular}

Finally, our approach was tested for the Brazilian system (System \#5) described by Carreno in [29]. The unique optimal solution evidenced by an exhaustive search accomplished throughout the landscape was successfully identified by EA plus BFSM-PA\#2 after 10840 evaluations: s7-s35-s51-s90-s96-s106-s118-s126-s135-s137s138-s141-s142-s144-s145-s146-147-s148-150-151-155.

The implemented method has exhibited good convergence for that solution corresponding to power losses of $280.1 \mathrm{~kW}$ as opposed to the initial configuration (s135-s136-s137-s138-s139-s140-s141-s142-s143-s144-s145s146-s147-s148-s149-150-s151-152-153-154-155) whose losses is $320.3 \mathrm{~kW}$.

\subsection{Multiobjective optimization}

The number of feasible solutions is disposed at the last column of Table 1 . It is important to mention that the paretooptimal solutions (PoS) was estimated for System \#4 through an extended run of NSGA-II set up for 20 million of evaluations (eval $\left.=2 \times 10^{7}\right)$ and population size of $2000($ npop=2000) believed by the authors to be satisfactory due to the size of the feasible space (see Table 1 ). As seen in Tables 4-7, the PoS attained by NSGA-II using BFSM-PA\#1 differs from the frontier achieved with BFSM-PA\#2 for Systems \#1 and \#2. However, PoS are kept the same when analyzing System \#3 under the same circumstances (Table 8 ). Near 96\% of solutions achieved

Table 6

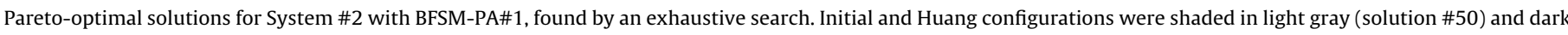
gray (solution \#10), respectively.

\begin{tabular}{|c|c|c|c|c|c|c|c|c|c|c|c|c|c|c|c|c|c|c|c|}
\hline \multirow[t]{2}{*}{ Solution } & \multicolumn{5}{|c|}{$\begin{array}{l}\text { Variable domain } \\
\text { (opened switches) }\end{array}$} & \multicolumn{4}{|c|}{ Objective domain } & \multirow[t]{2}{*}{ Solution } & \multicolumn{5}{|c|}{$\begin{array}{l}\text { Variable domain } \\
\text { (opened switches) }\end{array}$} & \multicolumn{4}{|c|}{ Objective domain } \\
\hline & $\mathrm{S} 1$ & $\mathrm{~S} 2$ & S3 & S4 & S5 & $\mathrm{PL}(\mathrm{kW})$ & VDI & CLI & NS & & S1 & $\mathrm{S} 2$ & S3 & S4 & S5 & $\mathrm{PL}(\mathrm{kW})$ & VDI & CLI & NS \\
\hline 1 & 13 & 20 & 59 & 62 & 70 & 94.0357 & 0.0131 & 0.2121 & 8 & 26 & 14 & 21 & 27 & 59 & 70 & 116.9494 & 0.0133 & 0.1747 & 8 \\
\hline 2 & 13 & 20 & 59 & 63 & 70 & 94.8489 & 0.0130 & 0.2085 & 8 & 27 & 14 & 21 & 59 & 62 & 70 & 100.4805 & 0.0125 & 0.1923 & 8 \\
\hline 3 & 13 & 20 & 59 & 65 & 70 & 107.9623 & 0.0120 & 0.1872 & 8 & 28 & 14 & 21 & 59 & 63 & 70 & 100.8507 & 0.0125 & 0.1900 & 8 \\
\hline 4 & 13 & 20 & 59 & 70 & 74 & 113.4621 & 0.0124 & 0.1823 & 6 & 29 & 14 & 21 & 59 & 65 & 70 & 110.9406 & 0.0120 & 0.1777 & 8 \\
\hline 5 & 13 & 21 & 59 & 62 & 70 & 94.0258 & 0.0130 & 0.2121 & 8 & 30 & 14 & 21 & 59 & 70 & 74 & 115.6911 & 0.0125 & 0.1751 & 6 \\
\hline 6 & 13 & 21 & 59 & 63 & 70 & 94.8399 & 0.0129 & 0.2085 & 8 & 31 & 14 & 59 & 62 & 70 & 71 & 98.5935 & 0.0141 & 0.2285 & 6 \\
\hline 7 & 13 & 21 & 59 & 65 & 70 & 107.9599 & 0.0120 & 0.1872 & 8 & 32 & 14 & 59 & 63 & 70 & 71 & 98.9570 & 0.0139 & 0.2238 & 6 \\
\hline 8 & 13 & 21 & 59 & 70 & 74 & 113.4615 & 0.0124 & 0.1824 & 6 & 33 & 14 & 59 & 65 & 70 & 71 & 109.5435 & 0.0125 & 0.1948 & 6 \\
\hline 9 & 13 & 59 & 65 & 70 & 71 & 109.5371 & 0.0126 & 0.1951 & 6 & 34 & 14 & 59 & 70 & 71 & 74 & 114.5668 & 0.0129 & 0.1879 & 4 \\
\hline 10 & 13 & 59 & 70 & 71 & 74 & 113.4058 & 0.0129 & 0.1860 & 4 & 35 & 17 & 27 & 46 & 59 & 70 & 124.3711 & 0.0161 & 0.1700 & 8 \\
\hline 11 & 14 & 16 & 59 & 63 & 70 & 110.2821 & 0.0153 & 0.1870 & 8 & 36 & 18 & 26 & 43 & 59 & 72 & 126.3141 & 0.0174 & 0.1689 & 8 \\
\hline 12 & 14 & 16 & 59 & 70 & 74 & 121.3372 & 0.0143 & 0.1720 & 6 & 37 & 18 & 26 & 59 & 70 & 72 & 123.6836 & 0.0161 & 0.1699 & 6 \\
\hline 13 & 14 & 17 & 27 & 59 & 70 & 120.4348 & 0.0144 & 0.1718 & 8 & 38 & 18 & 27 & 43 & 59 & 72 & 125.2246 & 0.0167 & 0.1691 & 8 \\
\hline 14 & 14 & 17 & 59 & 63 & 70 & 107.1823 & 0.0145 & 0.1872 & 8 & 39 & 18 & 27 & 46 & 59 & 70 & 124.4017 & 0.0161 & 0.1696 & 8 \\
\hline 15 & 14 & 17 & 59 & 65 & 70 & 115.1063 & 0.0134 & 0.1748 & 8 & 40 & 18 & 27 & 59 & 70 & 72 & 122.5501 & 0.0154 & 0.1701 & 6 \\
\hline 16 & 14 & 17 & 59 & 70 & 74 & 119.3066 & 0.0137 & 0.1722 & 6 & 41 & 18 & 43 & 59 & 65 & 72 & 120.3626 & 0.0159 & 0.1721 & 8 \\
\hline 17 & 14 & 18 & 27 & 59 & 70 & 118.4204 & 0.0139 & 0.1728 & 8 & 42 & 18 & 43 & 59 & 72 & 74 & 124.1857 & 0.0161 & 0.1695 & 6 \\
\hline 18 & 14 & 18 & 59 & 63 & 70 & 103.7524 & 0.0137 & 0.1881 & 8 & 43 & 18 & 46 & 59 & 70 & 74 & 123.3578 & 0.0154 & 0.1701 & 6 \\
\hline 19 & 14 & 18 & 59 & 65 & 70 & 112.7536 & 0.0128 & 0.1758 & 8 & 44 & 18 & 59 & 63 & 70 & 72 & 110.2842 & 0.0160 & 0.1855 & 6 \\
\hline 20 & 14 & 18 & 59 & 70 & 74 & 117.2275 & 0.0132 & 0.1733 & 6 & 45 & 18 & 59 & 65 & 70 & 72 & 117.4579 & 0.0146 & 0.1731 & 6 \\
\hline 21 & 14 & 20 & 27 & 59 & 70 & 116.9690 & 0.0134 & 0.1747 & 8 & 46 & 18 & 59 & 70 & 72 & 74 & 121.4671 & 0.0148 & 0.1705 & 4 \\
\hline 22 & 14 & 20 & 59 & 62 & 70 & 100.5263 & 0.0128 & 0.1922 & 8 & 47 & 19 & 59 & 63 & 70 & 72 & 110.3591 & 0.0160 & 0.1859 & 6 \\
\hline 23 & 14 & 20 & 59 & 63 & 70 & 100.8939 & 0.0128 & 0.1899 & 8 & 48 & 59 & 65 & 70 & 71 & 72 & 121.2523 & 0.0161 & 0.1846 & 4 \\
\hline 24 & 14 & 20 & 59 & 65 & 70 & 110.9658 & 0.0122 & 0.1777 & 8 & 49 & 59 & 70 & 71 & 72 & 74 & 124.0634 & 0.0160 & 0.1792 & 2 \\
\hline 25 & 14 & 20 & 59 & 70 & 74 & 115.7118 & 0.0126 & 0.1751 & 6 & 50 & 70 & 71 & 72 & 73 & 74 & 204.7992 & 0.0251 & 0.2201 & 0 \\
\hline
\end{tabular}


Table 7

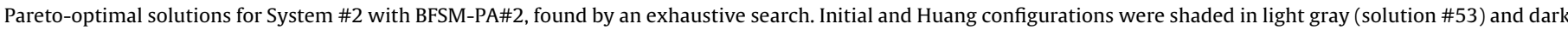
gray (solution \#10), respectively.

\begin{tabular}{|c|c|c|c|c|c|c|c|c|c|c|c|c|c|c|c|c|c|c|c|}
\hline \multirow[t]{2}{*}{ Solution } & \multicolumn{5}{|c|}{$\begin{array}{l}\text { Variable domain } \\
\text { (opened switches) }\end{array}$} & \multicolumn{4}{|c|}{ Objective domain } & \multirow[t]{2}{*}{ Solution } & \multicolumn{5}{|c|}{$\begin{array}{l}\text { Variable domain } \\
\text { (opened switches) }\end{array}$} & \multicolumn{4}{|c|}{ Objective domain } \\
\hline & $\mathrm{S} 1$ & $\mathrm{~S} 2$ & S3 & S4 & S5 & $\mathrm{PL}(\mathrm{kW})$ & VDI & CLI & NS & & $\mathrm{S} 1$ & $\mathrm{~S} 2$ & S3 & S4 & S5 & $\mathrm{PL}(\mathrm{kW})$ & VDI & CLI & NS \\
\hline 1 & 13 & 20 & 59 & 62 & 70 & 99.6735 & 0.0133 & 0.2173 & 8 & 28 & 14 & 21 & 59 & 63 & 70 & 106.9591 & 0.0127 & 0.1945 & 8 \\
\hline 2 & 13 & 20 & 59 & 63 & 70 & 100.7195 & 0.0133 & 0.2135 & 8 & 29 & 14 & 21 & 59 & 65 & 70 & 119.6241 & 0.0123 & 0.1828 & 8 \\
\hline 3 & 13 & 20 & 59 & 65 & 70 & 116.5636 & 0.0123 & 0.1924 & 8 & 30 & 14 & 21 & 59 & 70 & 74 & 125.3567 & 0.0128 & 0.1805 & 6 \\
\hline 4 & 13 & 20 & 59 & 70 & 74 & 123.0714 & 0.0128 & 0.1877 & 6 & 31 & 14 & 59 & 62 & 70 & 71 & 104.4965 & 0.0144 & 0.2342 & 6 \\
\hline 5 & 13 & 21 & 59 & 62 & 70 & 99.6629 & 0.0133 & 0.2173 & 8 & 32 & 14 & 59 & 63 & 70 & 71 & 105.0487 & 0.0142 & 0.2293 & 6 \\
\hline 6 & 13 & 21 & 59 & 63 & 70 & 100.7099 & 0.0132 & 0.2136 & 8 & 33 & 14 & 59 & 65 & 70 & 71 & 118.1894 & 0.0128 & 0.2001 & 6 \\
\hline 7 & 13 & 21 & 59 & 65 & 70 & 116.5612 & 0.0123 & 0.1924 & 8 & 34 & 14 & 59 & 70 & 71 & 74 & 124.2021 & 0.0132 & 0.1933 & 4 \\
\hline 8 & 13 & 21 & 59 & 70 & 74 & 123.0707 & 0.0127 & 0.1877 & 6 & 35 & 17 & 46 & 59 & 70 & 74 & 133.2759 & 0.0158 & 0.1760 & 6 \\
\hline 9 & 13 & 59 & 65 & 70 & 71 & 118.1852 & 0.0129 & 0.2004 & 6 & 36 & 18 & 26 & 43 & 59 & 72 & 136.7585 & 0.0178 & 0.1745 & 8 \\
\hline 10 & 13 & 59 & 70 & 71 & 74 & 124.1743 & 0.0132 & 0.1937 & 4 & 37 & 18 & 26 & 59 & 70 & 72 & 134.0463 & 0.0165 & 0.1755 & 6 \\
\hline 11 & 14 & 16 & 59 & 63 & 70 & 116.9634 & 0.0156 & 0.1918 & 8 & 38 & 18 & 27 & 43 & 59 & 72 & 135.4212 & 0.0171 & 0.1747 & 8 \\
\hline 12 & 14 & 16 & 59 & 65 & 70 & 126.2977 & 0.0143 & 0.1798 & 8 & 39 & 18 & 27 & 46 & 59 & 70 & 134.5805 & 0.0164 & 0.1752 & 8 \\
\hline 13 & 14 & 16 & 59 & 70 & 74 & 131.2090 & 0.0146 & 0.1774 & 6 & 40 & 18 & 27 & 59 & 70 & 72 & 132.6620 & 0.0158 & 0.1756 & 6 \\
\hline 14 & 14 & 17 & 27 & 59 & 70 & 130.4756 & 0.0148 & 0.1773 & 8 & 41 & 18 & 43 & 59 & 65 & 72 & 129.4040 & 0.0163 & 0.1774 & 8 \\
\hline 15 & 14 & 17 & 59 & 63 & 70 & 113.6739 & 0.0148 & 0.1919 & 8 & 42 & 18 & 43 & 59 & 72 & 74 & 134.1433 & 0.0165 & 0.1750 & 6 \\
\hline 16 & 14 & 17 & 59 & 65 & 70 & 123.9570 & 0.0137 & 0.1799 & 8 & 43 & 18 & 46 & 59 & 65 & 70 & 128.5366 & 0.0156 & 0.1779 & 8 \\
\hline 17 & 14 & 17 & 59 & 70 & 74 & 129.1022 & 0.0140 & 0.1776 & 6 & 44 & 18 & 46 & 59 & 70 & 74 & 133.2975 & 0.0158 & 0.1756 & 6 \\
\hline 18 & 14 & 18 & 27 & 59 & 70 & 128.3896 & 0.0142 & 0.1783 & 8 & 45 & 18 & 59 & 62 & 70 & 72 & 116.8439 & 0.0165 & 0.1927 & 6 \\
\hline 19 & 14 & 18 & 59 & 63 & 70 & 110.0358 & 0.0140 & 0.1927 & 8 & 46 & 18 & 59 & 63 & 70 & 72 & 116.9415 & 0.0163 & 0.1903 & 6 \\
\hline 20 & 14 & 18 & 59 & 65 & 70 & 121.5088 & 0.0131 & 0.1809 & 8 & 47 & 18 & 59 & 65 & 70 & 72 & 126.3968 & 0.0149 & 0.1783 & 6 \\
\hline 21 & 14 & 18 & 59 & 70 & 74 & 126.9473 & 0.0135 & 0.1786 & 6 & 48 & 18 & 59 & 70 & 72 & 74 & 131.3368 & 0.0151 & 0.1760 & 4 \\
\hline 22 & 14 & 20 & 59 & 62 & 70 & 106.4344 & 0.0130 & 0.1968 & 8 & 49 & 19 & 59 & 62 & 70 & 72 & 116.8942 & 0.0165 & 0.1931 & 6 \\
\hline 23 & 14 & 20 & 59 & 63 & 70 & 107.0049 & 0.0130 & 0.1944 & 8 & 50 & 19 & 59 & 63 & 70 & 72 & 117.0126 & 0.0163 & 0.1907 & 6 \\
\hline 24 & 14 & 20 & 59 & 65 & 70 & 119.6503 & 0.0124 & 0.1827 & 8 & 51 & 59 & 65 & 70 & 71 & 72 & 130.4130 & 0.0164 & 0.1901 & 4 \\
\hline 25 & 14 & 20 & 59 & 70 & 74 & 125.3781 & 0.0129 & 0.1804 & 6 & 52 & 59 & 70 & 71 & 72 & 74 & 134.0666 & 0.0163 & 0.1848 & 2 \\
\hline 26 & 14 & 21 & 27 & 59 & 70 & 126.8681 & 0.0136 & 0.1801 & 8 & 53 & 70 & 71 & 72 & 73 & 74 & 225.0028 & 0.0262 & 0.2289 & 0 \\
\hline 27 & 14 & 21 & 59 & 62 & 70 & 106.3857 & 0.0128 & 0.1968 & 8 & & & & & & & & & & \\
\hline
\end{tabular}

\section{Table 8}

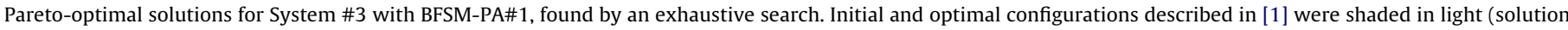
\#2) and dark gray (solution \#3), respectively.

\begin{tabular}{|c|c|c|c|c|c|c|c|}
\hline \multirow[t]{2}{*}{ Solution } & \multicolumn{3}{|c|}{ Variable domain (opened switches) } & \multicolumn{4}{|c|}{ Objective domain } \\
\hline & S1 & $\mathrm{S} 2$ & S3 & $\mathrm{PL}(\mathrm{kW})$ & VDI & CLI & NS \\
\hline 1 & 4 & 9 & 11 & 483.8689 & 0.0125 & 0.2616 & 2 \\
\hline 2 & 4 & 11 & 13 & 511.4355 & 0.0132 & 0.2601 & 0 \\
\hline 3 & 6 & 9 & 11 & 466.1267 & 0.0115 & 0.2611 & 4 \\
\hline 4 & 6 & 11 & 13 & 493.1542 & 0.0121 & 0.2597 & 2 \\
\hline
\end{tabular}

Table 9

Performance comparison among different codifications for System \#1 and System \#2.

\begin{tabular}{|c|c|c|c|c|c|c|c|c|c|}
\hline \multirow{2}{*}{$\begin{array}{l}\text { List of references } \\
\text { Codification }\end{array}$} & \multirow[b]{2}{*}{$\begin{array}{l}\text { Chrme. } \\
\text { length }\end{array}$} & \multicolumn{4}{|l|}{ System \#1 } & \multicolumn{4}{|l|}{ System \#2 } \\
\hline & & $\begin{array}{l}\text { Mean CPU } \\
\text { time (s) }\end{array}$ & $\begin{array}{l}\text { Average } \\
\text { cardinality of } \\
\text { Front \#1 }\end{array}$ & $\begin{array}{l}\text { Average } \\
\text { number of PoS } \\
\text { achieved }\end{array}$ & $\begin{array}{l}\text { Average } \\
\text { percentage of } \\
\text { PoS }(\%)\end{array}$ & $\begin{array}{l}\text { Mean CPU } \\
\text { time (s) }\end{array}$ & $\begin{array}{l}\text { Average } \\
\text { cardinality of } \\
\text { Front \#1 }\end{array}$ & $\begin{array}{l}\text { Average } \\
\text { number of } \\
\text { PoS achieved }\end{array}$ & $\begin{array}{l}\text { Average } \\
\text { percentage } \\
\text { of PoS (\%) }\end{array}$ \\
\hline Zhu [1] & $N_{W}$ & 17.5 & 13.8 & 12.2 & 32.1 & 37.8 & 13.8 & 8.2 & 16.4 \\
\hline Carreno [29] & $N_{L}$ & 18.5 & 11.4 & 3.8 & 10 & 40.2 & 11.4 & 2.0 & 4.0 \\
\hline Milani [35] & $N_{W}$ & 22.5 & 16.2 & 15 & 39.5 & 50.6 & 16.4 & 6.2 & 12.4 \\
\hline Mendoza with local elitism [37] & $N_{L}$ & 17.2 & 9.4 & 6.4 & 16.8 & 35.9 & 12.4 & 3.4 & 6.8 \\
\hline Mendoza without local elitism [37] & $N_{L}$ & 16.3 & 12.8 & 7.8 & 20.5 & 32.3 & 13.8 & 6.6 & 13.2 \\
\hline Proposed & $N_{W}$ & 16.4 & 15.0 & 12.2 & 32.1 & 36.3 & 14.2 & 8.2 & 16.4 \\
\hline
\end{tabular}

Table 10

Performance comparison among different codifications for System \#3 and System \#4.

\begin{tabular}{|c|c|c|c|c|c|c|c|c|c|}
\hline \multicolumn{2}{|l|}{ List of references } & \multicolumn{4}{|l|}{ System \#3 } & \multicolumn{4}{|l|}{ System \#4 } \\
\hline Codification & $\begin{array}{l}\text { Chrme. } \\
\text { length }\end{array}$ & $\begin{array}{l}\text { Mean }^{\dagger} \text { CPU } \\
\text { time }(s)\end{array}$ & $\begin{array}{l}\text { Average } \\
\text { cardinality of } \\
\text { front } \# 1\end{array}$ & $\begin{array}{l}\text { Average } \\
\text { number of PoS } \\
\text { achieved }\end{array}$ & $\begin{array}{l}\text { Average } \\
\text { percentage of } \\
\text { PoS }(\%)\end{array}$ & $\begin{array}{l}\text { Mean CPU } \\
\text { time (s) }\end{array}$ & $\begin{array}{l}\text { Average } \\
\text { cardinality of } \\
\text { Front \#1 }\end{array}$ & $\begin{array}{l}\text { Average } \\
\text { Pumber of } \\
\text { PoS achieved }\end{array}$ & $\begin{array}{l}\text { Average } \\
\text { percentage } \\
\text { of } \mathrm{PoS}(\%)\end{array}$ \\
\hline Zhu [1] & $N_{W}$ & 2.0 & 3.4 & 3.4 & 85.0 & 44.1 & 15.2 & 4.1 & 1.4 \\
\hline Carreno [29] & $N_{L}$ & 2.1 & 2.2 & 2.0 & 50.0 & 47.8 & 9.0 & 2.2 & 0.7 \\
\hline Milani [35] & $N_{W}$ & 2.4 & 3.2 & 3.2 & 80.0 & 69.9 & 13.3 & 3.5 & 1.2 \\
\hline Mendoza with local elitism [37] & $N_{L}$ & 2.0 & 1.6 & 1.6 & 40.0 & 41.7 & 10.9 & 1.5 & 0.5 \\
\hline Mendoza without local elitism [37] & $N_{L}$ & 1.9 & 2.4 & 2.2 & 55.0 & 38.1 & 12.6 & 2.8 & 0.9 \\
\hline Proposed & $N_{W}$ & 1.9 & 3.6 & 3.6 & 90.0 & 42.2 & 16.2 & 5.4 & 1.8 \\
\hline
\end{tabular}




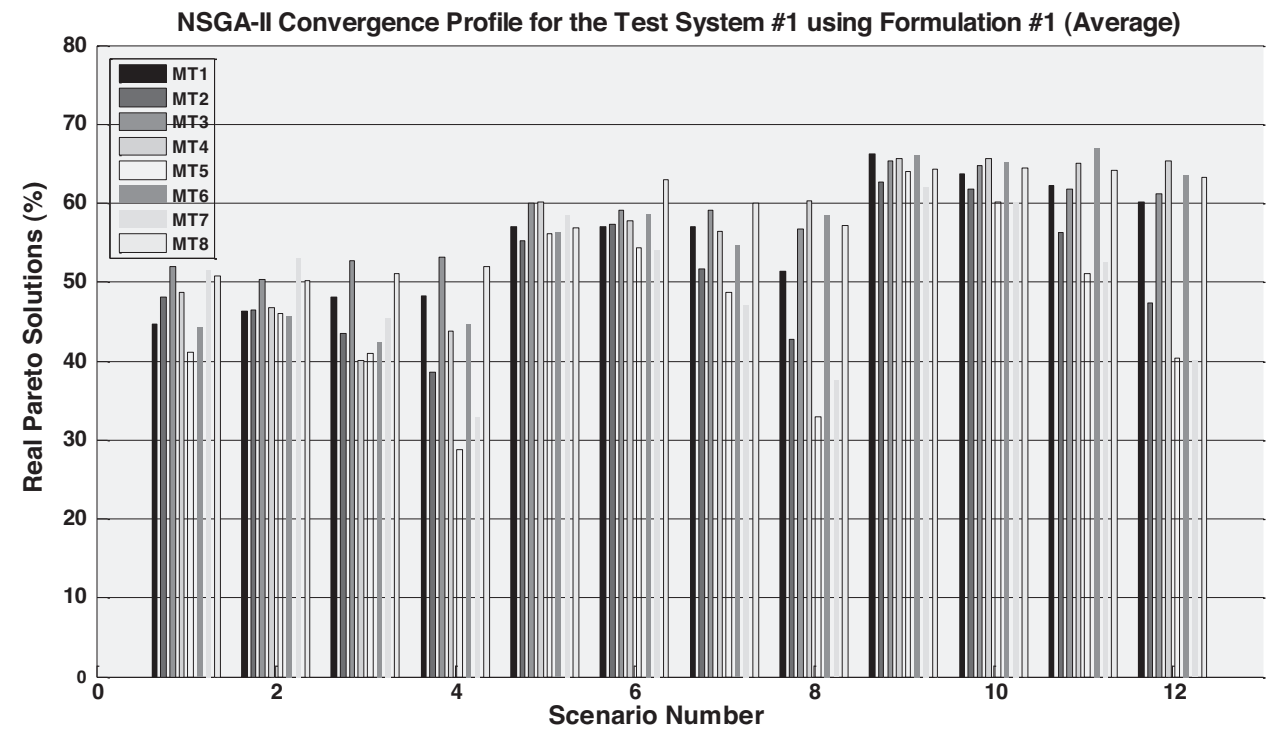

(a)

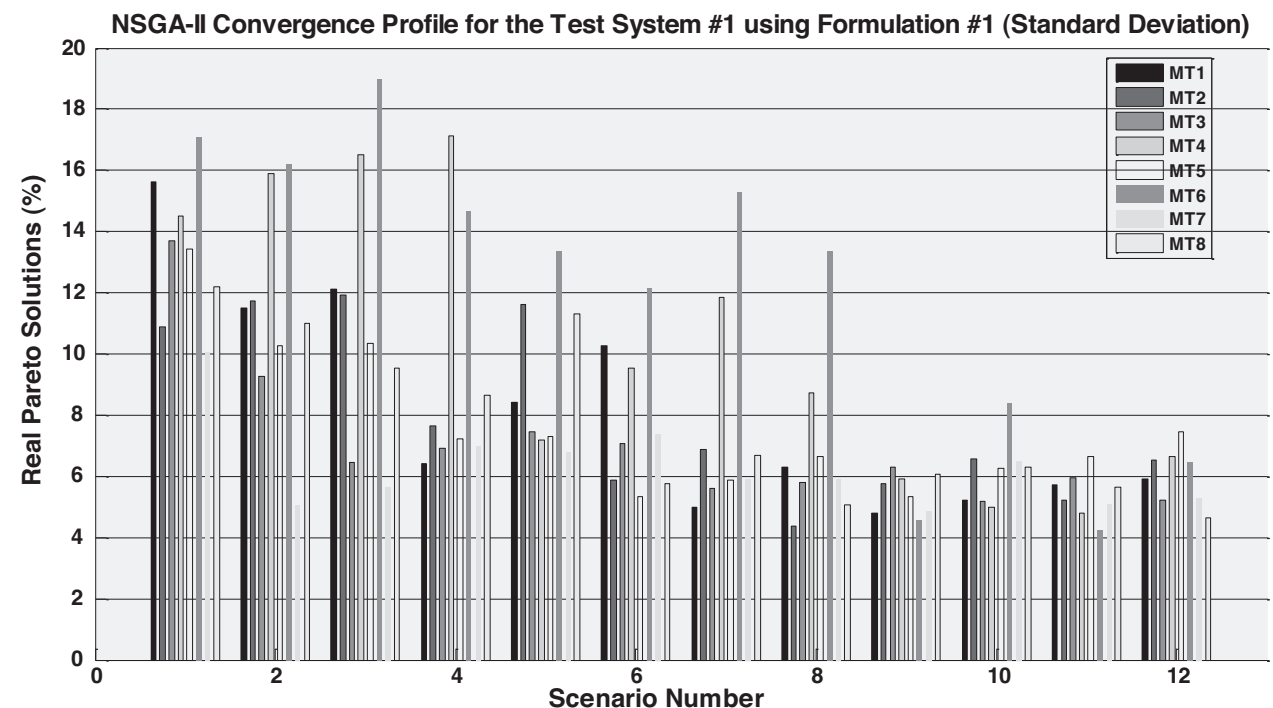

(b)

Fig. 6. NSGA-II convergence using eight different mutation patterns to solve System \#1: (a) average values and (b) standard deviation.

with BFSM-PA\#1 for System \#2 is found in the PoS obtained through BFSM-PA\#2. Still, PoS found with BFSM-PA\#2 has $90.6 \%$ of solutions coinciding with non-dominated found by BFSMPA\#1.
For System \#3, topologically feasible configurations represent almost $34 \%$ of the possibilities found with any three switches open and $0.30 \%$ of the whole search space. For a larger system such as System \#2, still small than real world distribution

Table 11

Inverse generational distance (IGD) of 12 scenarios for mutation type 1-8 related to systems 1-2. Best IGDs are shaded.

\begin{tabular}{|c|c|c|c|c|c|c|c|c|c|c|c|c|c|c|c|c|c|c|c|}
\hline \multirow[t]{2}{*}{ Scenario } & \multicolumn{8}{|c|}{ System \#1 } & \multirow[t]{2}{*}{ Best } & \multirow[t]{2}{*}{ Scenario } & \multicolumn{8}{|c|}{ System \#2 } & \multirow[t]{2}{*}{ Best } \\
\hline & MT1 & MT2 & MT3 & MT4 & MT5 & MT6 & MT7 & MT8 & & & MT1 & MT2 & MT3 & MT4 & MT5 & MT6 & MT7 & MT8 & \\
\hline 1 & 1.4048 & 1.3691 & 1.3541 & 1.5838 & 1.7778 & 1.8616 & 1.1279 & 1.4798 & MT7 & 1 & 1.0525 & 0.9812 & 0.9431 & 1.1021 & 1.1089 & 1.0817 & 1.0313 & 1.1748 & MT3 \\
\hline 2 & 1.7674 & 1.2235 & 1.3263 & 1.5183 & 1.2532 & 1.7313 & 1.0127 & 1.5005 & MT7 & 2 & 0.9913 & 1.1162 & 0.9553 & 1.1162 & 1.1402 & 1.0186 & 0.9492 & 1.0452 & MT7 \\
\hline 3 & 1.4770 & 1.3050 & 0.9889 & 2.0125 & 1.3844 & 2.0400 & 0.9226 & 1.2709 & MT7 & 3 & 1.0769 & 1.1839 & 0.9676 & 1.0927 & 1.2842 & 1.0343 & 0.9377 & 1.0169 & MT7 \\
\hline 4 & 1.2253 & 1.2536 & 0.8134 & 1.5804 & 1.5174 & 1.6920 & 1.1762 & 1.2279 & MT3 & 4 & 1.1276 & 1.3076 & 0.9751 & 1.1000 & 1.7487 & 1.0048 & 1.1416 & 1.0959 & MT3 \\
\hline 5 & 1.2237 & 1.3174 & 0.9828 & 1.4815 & 1.2597 & 1.4498 & 0.9857 & 1.1502 & MT3 & 5 & 0.7541 & 0.7701 & 0.6497 & 0.6977 & 0.7902 & 0.7434 & 0.6383 & 0.7423 & MT7 \\
\hline 6 & 1.3024 & 1.0362 & 0.8980 & 1.3910 & 1.1793 & 1.2060 & 0.9073 & 0.9308 & MT3 & 6 & 0.8520 & 0.7404 & 0.7611 & 0.6106 & 0.8730 & 0.7310 & 0.7626 & 0.7961 & MT4 \\
\hline 7 & 0.9672 & 1.2320 & 0.9198 & 1.4500 & 1.1810 & 1.2045 & 0.9250 & 1.2573 & MT3 & 7 & 0.7828 & 0.9717 & 0.8222 & 0.7903 & 1.0348 & 0.6500 & 0.8087 & 0.7415 & MT6 \\
\hline 8 & 1.2449 & 1.1380 & 0.7637 & 1.2369 & 1.3737 & 1.3066 & 1.0959 & 1.0637 & MT3 & 8 & 0.7759 & 1.1322 & 0.8020 & 0.7140 & 1.5131 & 0.6152 & 0.9932 & 0.7669 & MT6 \\
\hline 9 & 0.8196 & 0.7012 & 0.8865 & 0.9174 & 0.7915 & 0.9828 & 0.7125 & 0.8895 & MT5 & 9 & 0.4787 & 0.5585 & 0.5734 & 0.4667 & 0.5815 & 0.4089 & 0.5599 & 0.5163 & MT6 \\
\hline 10 & 0.8408 & 0.7697 & 0.8958 & 0.7275 & 0.6820 & 1.0678 & 0.7284 & 0.8252 & MT5 & 10 & 0.5154 & 0.6359 & 0.5189 & 0.5347 & 0.6613 & 0.4798 & 0.5725 & 0.4968 & MT6 \\
\hline 11 & 0.7724 & 0.8902 & 0.7913 & 0.9661 & 1.0313 & 0.8000 & 0.8091 & 0.6403 & MT8 & 11 & 0.5420 & 0.7244 & 0.5734 & 0.3945 & 0.8618 & 0.5064 & 0.6047 & 0.5913 & MT4 \\
\hline 12 & 0.9016 & 0.9311 & 0.6043 & 0.7676 & 1.1256 & 0.8936 & 0.9556 & 0.6265 & MT3 & 12 & 0.6928 & 0.8923 & 0.6219 & 0.5319 & 1.1990 & 0.4901 & 0.7645 & 0.4885 & MT8 \\
\hline
\end{tabular}




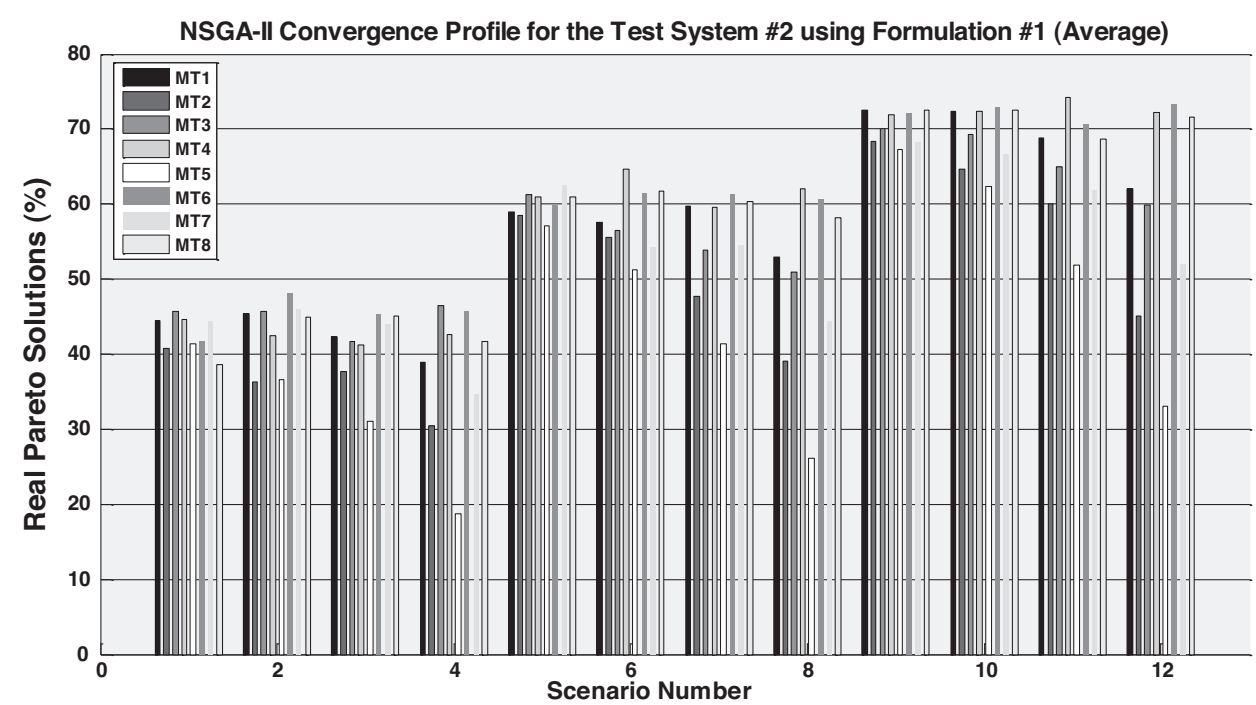

(a)

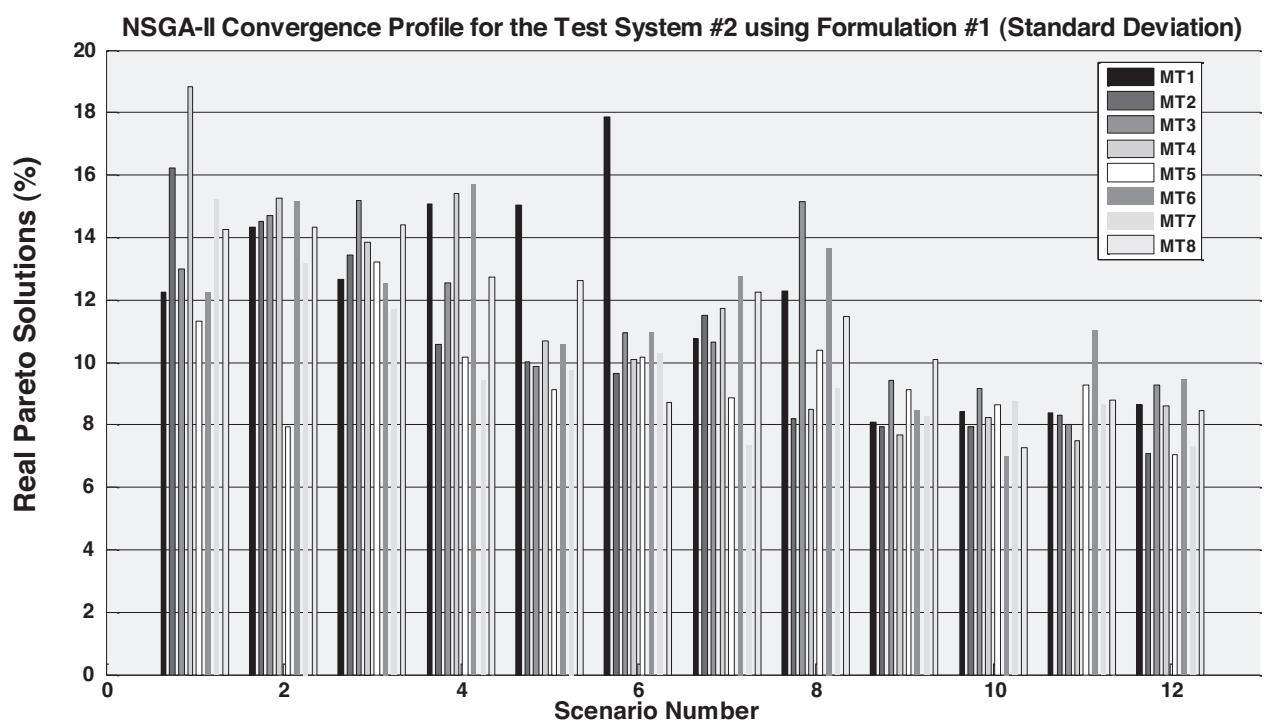

(b)

Fig. 7. NSGA-II convergence using eight different mutation patterns to solve System \#2: (a) average values and (b) standard deviation.

networks, the percentage of allowable configurations reaches $0.0389 \%(376,028 / 966,525,840)$ or $1.99 \mathrm{E}-15 \%$, admitting all binary possibilities seen in Table 1.

A PoS of 303 optima was found for System \#4 using BFSM-PA\#1 and 290 optima were found when BFSM-PA\#2 is considered. Due to the search space extensiveness (see Table 1), the exhaustive search was not used to find PoS avoiding prohibitive computation time. As already mentioned, NSGA-II was executed for $n p o p=2000$ and 10,000 generations, totalizing 20 million of evaluations which was considered enough to properly estimate PoS in System \#4. This PoS is not presented here due to a matter of space.

\subsection{Codification performance}

To validate our proposition, we choose three criteria namely CPU time, cardinality of the first frontier, and number of global optima achieved. Since we carried out five executions for each coding scheme, those criteria are average values indeed. All networks were tested for BFSM-PA\#1 with crossover and mutation rates of 0.98 and 0.05 , respectively. A population of 20 chromosomes over 60 generations was set up for the NSGA-II and results for Systems
$\# 1, \# 2, \# 3$, and \#4 are presented in Tables 9 and 10. Because System \#3 is smaller, npop was set up to 10 and eval to 30. Respective results are shown in Table 9. The time spent in the loop detection procedure used by Mendoza codification scheme is not accounted on CPU time showed in Tables 9 and 10.

The local search step associated with Carreno approach [29] was replaced by the mutation procedure that best fitted among the ones presented in Section 4.3 to allow a fair comparison. From Table 9, it seems that our coding scheme achieved acceptable stability showing good convergence for both systems. On the other hand, the scheme from Milani [35] has attained the greatest percentage of solutions for System \#1, but its performance was not kept to System \#2 for which either Zhu codification as our implementation have achieved better results. Convergence characteristics are also shown in Table 10 for Systems \#3 and \#4.

\subsection{Evaluation of the proposed operators}

The NSGA-II was adjusted to npop equals to 40 and number of generations equals to 100 (neval $=4000$ ) for different adjustments of $m r$ and $c r$ according to the sequenced combinations given by the 


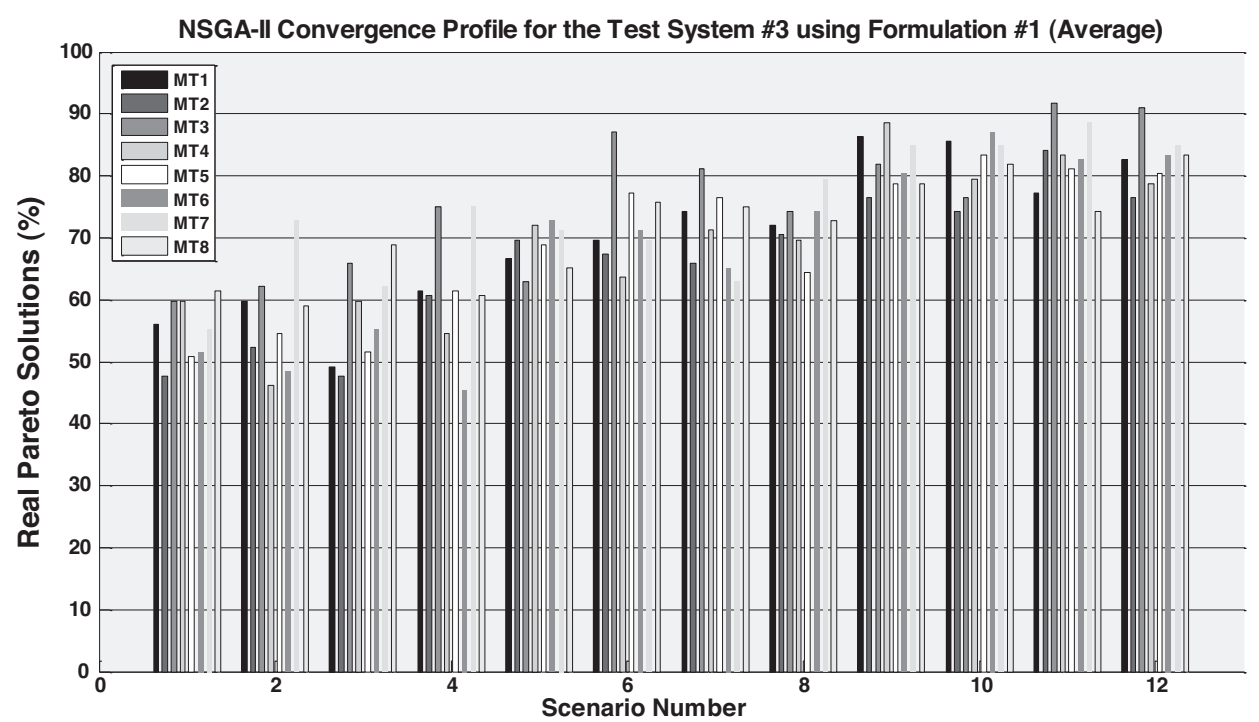

(a)

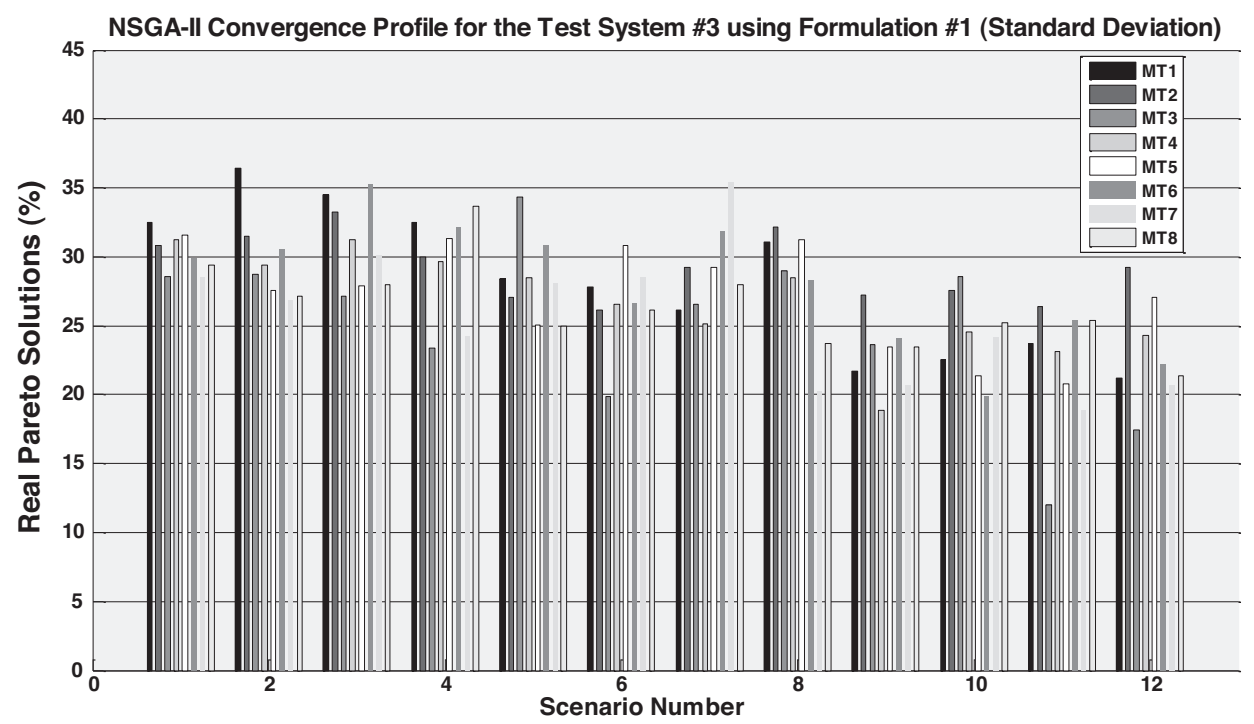

(b)

Fig. 8. NSGA-II convergence using eight different mutation patterns to solve System \#3: (a) average values and (b) standard deviation.

niched loops shown in Listing 1 . On those combinations, three values for the crossover rate and four values of mutation rate covered twelve probing scenarios. In Fig. 6(a) and (b), convergence performance is presented for eight types of mutation already explained in Section 4.3.

Listing 1 Rate variation for crossover and mutation totalizing 12 scenarios. Parameters neval and npop have predefined values.

\begin{tabular}{|c|c|}
\hline 1 & For each $c r:=\{0.5,0.75,1.0\}$ \\
\hline 2 & For each $m r:=\{0.05,0.1,0.2,0.4\}$ \\
\hline 3 & execute NSGA-II (eval, npop, $c r, m r$ ) \\
\hline 4 & End \\
\hline 5 & End \\
\hline
\end{tabular}

Fig. 6a presents average values $(m)$ for the percentage of PoS attained and Fig. $6 \mathrm{~b}$ its deviation $(\Delta)$, both calculated for 33 trials. It is clear that scenarios 9-12 exhibited the best results, in which mutation types $1,3,4$, and 6 have excelled if compared to the remaining ones. System \#2 has experienced convergence for eight mutation types after 33 runs, shown in Fig. 7(a) and (b). The
NSGA-II was adjusted to npop $=60$ and eval $=6000$. Rate values $m r$ and $c r$ were given according to Listing 1 . Convergence of the algorithm is showed for System \#3 in Fig. 8(a) and (b) for which NSGA-II was adjusted to npop $=20$ and eval $=200$ in scenarios also described by Listing 1 .

From Figs. 6-8, mutation types 3 and 7 were the best adaption to DFR problem achieving higher percentage of PoS with lower deviation in most scenarios of Systems \#1 and \#2. Table 11 shows the mean values of Inverse Generational Distance (IGD) metric for those systems in case of each scenario (per row) and mutation (per column). Smaller IGDs correspond to better convergence of the evolutionary algorithm. From those values, it is clear that MT3 and MT7 are good choices. In Table 11, the lowest values achieved by each mutation type are shaded in gray. It is also notable those values are attained for higher crossover rates. In most of the scenarios, MT7 had an acceptable performance for small values of crossover and mutation rates. It is clear from Table 11 that minor values are located at the latest scenarios (bottom of Table 11). Experimental results showed that relativity among feasible solutions were not hold according to dominance ranking for the two different sets of equations (BFSM-PA\#1 and BFSM-PA\#2) employed by the same 
power flow method on the same distribution system, as stated in [17]. The first approach is faster since it neglects power losses during iterations and power sum up can be performed one time if loads are modeled as constant power sinks. Depending on the tested system, relative accuracy is rather affected by the simplified assumptions presented in Huang's formulation.

\section{Conclusion}

Our approach has proved to be a promising coding scheme if it is compared to four other ones already proposed in the literature. Its strength lies on the flexibility in reaching a considerable amount of optima for a multi-objective formulation of the DFR problem, while requiring minimal memory space and computation time. It is worth to mention that LCA is extremely simple to implement and no major modifications must be carried out in NSGA-II implementation or other evolutionary algorithm, excepting on the decoding scheme to able correct individual evaluation. The proposed approach dispenses any supplemental information about loops or graph connectivity and it caters single source as well as multiple sources systems which is a major contribution of this work. There is no need for preprocessing while other strategies require loop detection or connectivity analysis. Distinctly from other approaches, our strategy never evaluates unfeasible solutions, once they are completely avoided by means of the coding and interpretation procedures. Exhaustive-like strategies are not employed and genetic operators are kept as simple as possible according to natural evolution paradigms. It was verified that the insertion of initial configuration in the first population of the evolutionary algorithm is needless since its lack does not degrade convergence. Finally, it was demonstrated through an extended convergence benchmarking that our coding scheme applied into NSGA-II has succeeded to five systems, for single or multiple sources, when other proposed approaches exhibited degradable convergence. Experiments have shown that our scheme has allowed the optimization procedure to reach global optima steadily throughout the search space, since it complies with important codification properties such as feasibility, locality, coverage, easy representativeness and bias, even though not being as fast as other schemes discussed in this article.

\section{Acknowledgement}

Authors would like to thank Brazilian agencies CAPES and CNPq for financial support.

\section{Appendix A.}

\section{List of symbols}

$N_{R} \quad$ number of source nodes

$N_{B} \quad$ number of total busses including sources

$N_{L} \quad$ number of lines

$N_{W} \quad$ number of sectionalizing and tie-switches

$\boldsymbol{W} \quad$ set of system switches

$\omega$ indexes of closed switches for a given configuration

$\overline{\boldsymbol{\omega}} \quad$ indexes of open switches for a given configuration

B set of busses, including load and source busses

$\boldsymbol{L} \quad$ set of distribution lines

$\Psi \quad$ set of switchless distribution lines

$\boldsymbol{\Omega} \quad$ set of switchable distribution lines

$\tau \quad$ set of powered distribution lines

$\bar{\tau} \quad$ set of distribution lines powered down

$k \quad$ bus iterator to denote a single element from $\boldsymbol{B}$

$i \quad$ line iterator to denote a single element from $\boldsymbol{L}$

$q \quad$ switch iterator to denote a single element from $\boldsymbol{W}$
$Z_{i} \quad$ line longitudinal impedance of line $i$

$V_{k}^{\min } \quad$ minimal bus at the $k$ th bus

$V_{k}^{k}$ nominal bus voltage at the $k$ th bus

$I_{i}{ }^{k} \quad$ current flow in $i$ th line

$I_{i}^{\max } \quad$ admissible current flow for line $i$

$\stackrel{S}{r}$ max $_{P_{k}} \quad$ total power capacity of the source $r$

$P_{k} \quad$ real power demand at bus $k$

$Q_{k} \quad$ reactive power demand at bus $k$

$N L \quad$ number of fundamental loops in the network

NT total number of valid trees (equal to the number of sources)

$s w_{q}^{c} \quad$ status of switch $q$ in configuration $c$ : open (0) and closed (1)

$\mathrm{cr} \quad$ crossover rate

$m r \quad$ mutation rate

nрор population size

eval number of evaluations

$\operatorname{Re}(\cdot) \quad$ real component of the argument

$\operatorname{Im}(\cdot) \quad$ imaginary component of the argument

$|\cdot| \quad$ argument modulus

\section{References}

[1] J.Z. Zhu, Optimal reconfiguration of electrical distribution network using the refined genetic algorithm, Electric Power Systems Research 62 (2002) 37-42.

[2] Z.Q. Zhou, D. Shirmohammadi, -H.E.W. Liu, Distribution feeder reconfiguration for service restoration and load balancing, IEEE Transactions on Power Systems 12 (May 2) (1997) 724-729.

[3] C.-T. Su, C.-S. Lee, Network reconfiguration of distribution systems using improved mixed-integer hybrid differential evolution, IEEE Transactions on Power Delivery 18 (July 3) (2003) 1022-1027.

[4] D.S. Popovic, R.M. Ciric, A multi-objective algorithm for distribution networks restoration, IEEE Transactions on Power Delivery 14 (July 3)(1999) 1134-1141.

[5] S. Toune, H. Fudo, T. Genji, Y. Fukuyama, Y. Nakanishi, Comparative study of modern heuristic algorithms to service restoration in distribution systems, IEEE Transactions on Power Delivery 17 (January 1) (2002) 173-181.

[6] T.E. McDermott, I. Drezga, R.P. Broadwater, A heuristic nonlinear constructive method for distribution system reconfiguration, IEEE Transactions on Power Systems 14 (May 2) (1999) 478-483.

[7] L.M. Queiroz, C. Lyra, Adaptive hybrid genetic algorithm for technical loss reduction in distribution networks under variable demands, IEEE Transactions on Power Systems 24 (February 1) (2009) 445-453.

[8] H.M. Braz, B.A. Souza, Distribution network reconfiguration using genetic algorithms with sequential encoding: subtractive and additive approaches, IEEE Transactions on Power Systems 26 (May 2) (2011) 582-593.

[9] G.R. Raidl, B.A. Julstrom, Edge sets: an effective evolutionary coding of spanning trees, IEEE Transactions on Evolutionary Computation 7 (June 3) (2003) 225-239.

[10] K. Deb, A. Pratap, S. Agarwal, T. Meyarivan, A fast and elitist multiobjective genetic algorithm: NSGA-II, IEEE Transactions on Evolutionary Computation 6 (April 2) (2002) 182-197.

[11] R.J. Sarfi, M.M.A. Salama, A.Y. Chikhani, A survey of the state of the art in distribution system reconfiguration for system loss reduction, Electric Power Systems Research 31 (1994) 61-70.

[12] A. Merlin, H. Back, Search for a minimal loss operating spanning tree configuration for an urban power distribution system, in: Proceedings on 5th Power Syst. Comp. Conf., PSCC, Cambridge, 1975.

[13] S. Civanlar, J.J. Grainger, H. Yin, S.S.H. Lee, Distribution feeder reconfiguration for loss reduction, IEEE Transactions on Power Delivery 3 (July 3) (1988) 1217-1223.

[14] M.E. Baran, F.F. Wu, Network reconfiguration in distribution systems for loss reduction and load balancing, IEEE Transactions on Power Delivery 4 (April 2) (1989) 1401-1407.

[15] D. Shirmohammadi, H.W. Hong, Reconfiguration of electric distribution networks for resistive line losses reduction, IEEE Transactions on Power Delivery 4 (April 2) (1989) 1492-1498.

[16] H.D. Chiang, R.J. Jumeau, Optimal network reconfigurations in distribution systems. Part 2 - Solution algorithms and numerical results, IEEE Transactions on Power Delivery 5 (July 3) (1990) 1568-1574.

[17] S.K. Goswami, S.K. Basu, A new algorithm for the reconfiguration of distribution feeders for loss minimization, IEEE Transactions on Power Delivery 7 (July 3) (1992) 1484-1491.

[18] J.A. Martin, A.J. Gil, A new heuristic approach for distribution systems loss reduction, Electric Power Systems Research 78 (2008) 1953-1958.

[19] J. Zhu, et al., A rule based comprehensive approach for reconfiguration, Electric Power Systems Research 79 (2009) 311-315.

[20] A.R. Abul'Wafa, A new heuristic approach for optimal reconfiguration in distribution systems, Electric Power Systems Research 81 (2011) 282-289. 
[21] A.J.G. Mena, J.A.M. Garcia, An efficient heuristic algorithm for reconfiguration based on branch power flows direction, Electric Power Systems Research 41 (2012) 71-75.

[22] K. Nara, A. Shiose, M. Kitagawa, T. Ishihara, Implementation of genetic algorithm for distribution systems loss minimum re-configuration, IEEE Transactions on Power Systems 7 (August 3) (1992) 1044-1051.

[23] Y.T. Hsiao, C.Y. Chien, Enhancement of restoration service in distribution systems using a combination fuzzy-GA method, IEEE Transactions on Power Systems 15 (November 4) (2000) 1394-1400.

[24] F. Rothlauf, On the bias and performance of the edge-set encoding, IEEE Transactions on Evolutionary Computation 13 (June (3)) (2009) 486-499.

[25] A. Swarnkar, N. Gupta, K.R. Niazi, A novel codification for meta-heuristic techniques used in distribution network, Electric Power Systems Research 81 (2011) 1619-1626.

[26] A.L. Morelato, A. Monticelli, Heuristic search approach to distribution system restoration, IEEE Transactions on Power Delivery 4 (October 4) (1989) 2235-2241.

[27] E.R. Ramos, A.G. Expósito, J.R. Santos, F.L. Iborra, Path-based distribution network modeling: application to reconfiguration for loss reduction, IEEE Transactions on Power Systems 20 (May 2) (2005) 556-564.

[28] B. Enacheanu, et al., Radial network reconfiguration using genetic algorithm based on the matroid theory, IEEE Transactions on Power Systems 23 (February 1) (2008) 186-195.

[29] E.M. Carreno, R. Romero, A. Padilha-Feltrin, An efficient codification to solve distribution network reconfiguration for loss reduction problem, IEEE Transactions on Power Systems 23 (November 4) (2008) 1542-1551.

[30] D.J. Shin, J. Kim, T.K. Kim, J.B. Choo, C. Singh, Optimal service restoration and reconfiguration of network using genetic-Tabu algorithm, Electric Power Systems Research 71 (2004) 145-152.

[31] Y.C. Huang, Enhanced genetic algorithm-based fuzzy multi-objective approach to distribution network reconfiguration, IEEE Proceedings: Generation, Transmission and Distribution 149 (September 5) (2002) 615-620.

[32] Y. Kumar, B. Das, J. Sharma, Service restoration in distribution system using non-dominated sorting genetic algorithm, Electric Power Systems Research 76 (2006) 768-777.

[33] Y. Fukuyama, H.D. Chiang, K.N. Miu, Parallel genetic algorithm for service restoration in electric power distribution systems, International Journal of Electrical Power and Energy Systems 18 (2) (1996) 111-119.

[34] V. Miranda, J.V. Ranito, L.M. Proença, Genetic algorithms in optimal multistage distribution network planning, IEEE Transactions on Power Systems 9 (November 4) (1994) 1927-1933.

[35] A.E. Milani, M.R. Haghifam, A new probabilistic approach for distribution network reconfiguration: applicability to real networks, Mathematical and Computer Modelling (June) (2011) 1-11.

[36] W.M. Lin, F.S. Cheng, M.T. Tsay, Distribution feeder reconfiguration with refined genetic algorithm, IEEE Proceedings: Generation, Transmission and Distribution 147 (November 6) (2000) 349-354.

[37] J. Mendoza, et al., Minimal loss reconfiguration using genetic algorithms with restricted population and addressed operators: real application, IEEE Transactions on Power Systems 21 (May 2) (2006) 948-954.

[38] A.C.B. Delbem, A.C.F. Carvalho, N.G. Bretas, Main chain representation for evolutionary algorithms applied to distribution system reconfiguration, IEEE Transactions on Power Systems 20 (February 1) (2005) 425-436.

[39] E. Lopez, H. Opazo, L. Garcia, P. Bastard, Online reconfiguration considering variability demand: applications to real networks, IEEE Transactions on Power Systems 19 (February 1) (2004) 549-553.
[40] V.J. Garcia, P.M. França, Multiobjective service restoration in electric distribution networks using a local search based heuristic, European Journal of Operational Research (2008) 694-705.

[41] J.B. Kruskal, On the shortest spanning subtree of a graph and the traveling salesman problem, Proceedings of the American Mathematical Society 7 (1) (1956) 48-50.

[42] R. Prim, Shortest connection networks and some generalisations, Bell System Technical Journal 36 (1957) 1389-1401.

[43] R. Ahuja, T. Magnanti, J. Orlin, Network Flows: Theory, Algorithms and Applications, Prentice Hall, Englewood Cliffs, 1993.

[44] D.E. Goldberg, Genetic Algorithms in Search, Optimization and Machine Learning, 1st ed., Addison-Wesley, Boston, MA, 1989.

[45] D. Shirmohammadi, H.W. Hong, A. Semlyen, G.X. Luo, A compensationbased power flow method for weakly meshed distribution and transmission networks, IEEE Transactions on Power Systems 3 (May 2) (1988) 753-762.

[46] A.C. Santos, A.C. Delbem, J.B. London Jr., N.G. Bretas, Node-depth encoding and multiobjective evolutionary algorithm applied to large-scale distribution system reconfiguration, IEEE Transactions on Power Systems 25 (August 3) (2010) 1254-1265.

[47] Y.Y. Hong, S.Y. Ho, Determination of network configuration considering multiobjective in distribution systems using genetic algorithms, IEEE Transactions on Power Systems 20 (May 2) (2005) 1062-1069.

[48] F.V. Gomes, et al., A new distribution system reconfiguration approach using optimum power flow and sensitivity analysis for loss reduction, IEEE Transactions on Power Systems 21 (November 4) (2006) 1616-1623.

[49] A.B. Morton, I.Y. Mareels, An efficient brute-force solution to the network reconfiguration problem, IEEE Transactions on Power Delivery 15 (July 3) (2000) $996-1000$.

[50] J.S. Savier, D. Das, Loss allocation to consumers before and after reconfiguration of radial distribution networks, International Journal of Electrical Power and Energy Systems 33 (2011) 540-549.

Carlos Henrique Nogueira de Resende Barbosa received his B.Sc. degree in Electrical Engineering from Universidade Federal de Minas Gerais (UFMG), Belo Horizonte, Brazil, in 1999. In 2002, he obtained his M.Sc. degree also from UFMG. Currently, he is a professor at Universidade Federal de Ouro Preto and he is pursuing the Ph.D. degree at UFMG. His research interests are electrical network optimization, multi-objective optimization, and fuzzy systems.

Rafael Frederico Alexandre received his B.Sc. degree on computing science in 2003 from Pontifícia Universidade Católica de Minas Gerais. He received his specialist degree in Software Engineer in 2005 from Universidade Federal de Minas Gerais (UFMG) and his M.Sc. degree in Electrical Engineering in 2009 from UFMG also. He is currently a professor at Universidade Federal de Ouro Preto and he is pursuing the Ph.D. degree at UFMG. His research interests include algorithms and data structures, multi-objective optimization and operational research.

João Antônio de Vasconcelos received the B.Sc. degree in electrical engineering from the Universidade Federal de Minas Gerais (UFMG), Belo Horizonte, Brazil, in 1982 the M.Sc. degree in electrical engineering from Universidade Federal da Paraíba in 1985, and the Ph.D. degree in electrical engineering from the École Centrale de Lyon, France, in 1994. Currently, he is a Senior Professor at Electrical Engineering Department in UFMG. Most of his research work includes optimization on design, maintenance and operation of systems, applied electromagnetic theory, and decision making. 